BI-TP 2004/40

May 2005

\title{
Scaling and Goldstone effects in a QCD with two flavours of adjoint quarks
}

\author{
J. Engels, S. Holtmann and T. Schulze \\ Fakultät für Physik, Universität Bielefeld, D-33615 Bielefeld, Germany
}

\begin{abstract}
We study QCD with two Dirac fermions in the adjoint representation at finite temperature by Monte Carlo simulations. In such a theory the deconfinement and chiral phase transitions occur at different temperatures. We locate the second order chiral transition point at $\beta_{c}=5.624(2)$ and show that the scaling behaviour of the chiral condensate in the vicinity of $\beta_{c}$ is in full agreeement with that of the $3 d O(2)$ universality class, and to a smaller extent comparable to the $3 d O(6)$ class. From the previously determined first order deconfinement transition point $\beta_{d}=5.236(3)$ and the two-loop beta function we find the ratio $T_{c} / T_{d} \approx 7.8(2)$. In the region between the two phase transitions we explicitly confirm the quark mass dependence of the chiral condensate which is expected due to the existence of Goldstone modes like in $3 d O(N)$ spin models. At the deconfinement transition $\langle\bar{\psi} \psi\rangle$ shows a gap, and below $\beta_{d}$, it is nearly mass-independent for fixed $\beta$.
\end{abstract}

PACS : 11.15.Ha; 12.38.Gc; 12.38.Aw; 75.10.Hk

Keywords: QCD thermodynamics; Chiral phase transition; $O(N)$ spin models

E-mail: engels, holtmann, tschulze@physik.uni-bielefeld.de 


\section{Introduction}

The classification of the chiral transition in finite temperature quantum chromodynamics (QCD) for two flavours of quarks is a long-standing problem. This refers in particular to analyses of lattice data obtained from simulations with staggered fermions. For the continuum theory a second order chiral transition is predicted to belong to the universality class of the three-dimensional $O(4)$ spin model $[1,2,3]$. Due to the lattice formulation of the fermionic sector of the theory, chiral symmetry is lost for Wilson fermions and reduced to an $O(2)$ symmetry for staggered fermions. Full symmetry is then recovered only in the continuum limit. Surprisingly, corresponding tests with Wilson fermions $[4,5]$ confirmed the proposed universality class, whereas the first tests with staggered fermions and the standard QCD action remained unsatisfactory [6]-[10]. Essentially, the comparison of universal critical quantities from $O(N)$ spin models to lattice QCD data failed in the close vicinity of the chiral transition. However, at temperatures above the transition some agreement was found on the pseudocritical line, namely for the form of the line itself and for finite size scaling of the chiral condensate on the line [11]. The apparent failure of the tests was attributed to a very small critical region, i. e. use of too large $m_{q} a-$ values and lattice artifacts, i. e. calculations with too small $N_{\tau}$ and/or unimproved actions.

The issue is still under consideration and still unsettled: Kogut and Sinclair, who use a QCD action including an irrelevant 4-fermion term, conclude from simulations at $m_{q}=0$ on a $24^{3} \times 8$ lattice [12] that the critical region must be very small, but the transition class could be compatible to the $3 d O(2) / O(4)$ universality class. Chandrasekharan and Strouthos [13] performed simulations at strong coupling and compared their data to predictions from chiral perturbation theory for $O(2)$. They find full agreement for masses which are much smaller than those normally used in QCD calculations. Contrary to that, D' Elia et al. [14] exclude $O(2) / O(4)$ scaling in their finite-size-scaling analysis of specific heat and susceptibility data for the standard QCD action. Instead they find consistency of their data with a first order transition.

The difficulties and discrepancies encountered in the investigation of the nature of the chiral transition could be related to another puzzle of finite temperature QCD: the coincidence of the chiral and the deconfinement transitions. Though there is no a priori reason why the two transitions should occur at the same temperature in QCD, they seem to be strongly bound to each other. The interplay of the deconfinement and chiral transitions could then obscure the critical chiral behaviour in the vicinity of the common critical point. It is possible to study the properties of the chiral transition without direct interference from the deconfinement transition. In a special QCD-related model, $S U\left(N_{c}\right)$ gauge theory with two Dirac fermions in the adjoint representation (aQCD), the deconfinement transition occurs at a temperature $T_{d}$ which is definitely smaller than $T_{c}$, the chiral transition temperature $[15,16]$. 
In general, aQCD with $N_{f}$ massless fermions exhibits an $S U\left(2 N_{f}\right)$ chiral symmetry, which is spontaneously broken at low temperatures to $S O\left(2 N_{f}\right)[17,18]$. Since, in contrast to QCD, the fermionic part of the aQCD action does not break the global $Z\left(N_{c}\right)$ centre symmetry of the $S U\left(N_{c}\right)$ gauge part, the model holds in addition the same global $Z\left(N_{c}\right)$ symmetry as pure $S U\left(N_{c}\right)$ gauge theory. Correspondingly, one expects two finite temperature transitions and there are two independent order parameters, the Polyakov loop and the chiral condensate, which indicate the breaking of the respective symmetries. The universality class of the chiral transition of aQCD is normally different from that of QCD. For two flavours of adjoint Dirac fermions the chiral symmetry group is $S U(4)$. That group is isomorphic to $O(6)$, which breaks to $O(5)$, implying the existence of 5 Goldstone bosons. The breaking $S U(4) \rightarrow S O(4)$ leads however to 9 Goldstone bosons. The corresponding transition belongs therefore to a different universality class. In a recent paper [19] this issue has been investigated using renormalization-group arguments and first results for the critical exponents of this class have been obtained: $\eta \approx 0.2, \nu \approx 1.1$. The points we have just raised pertain to continuum theory. On the lattice, the staggered formulation leaves, like in QCD, only a remaining $O(2)$ chiral symmetry. In the continuum limit one should however recover the full $S U(4)$ symmetry and the critical behaviour following from its breaking to $S O(4)$.

The main objective of this paper is to investigate the chiral transition of aQCD and if possible to establish its universality class. Since we are simulating aQCD on the lattice with two flavours of staggered Dirac fermions ${ }^{1}$ we expect to find the three-dimensional $O(2)$ class. The thermodynamics of aQCD with $N_{c}=3$ has been studied to a great extent already by Karsch and Lütgemeier [16]. In particular, a first order deconfinement transition was found at $\beta_{d}=5.236(3)$ and a second order chiral transition at $\beta_{c}=5.79(5)$ was inferred from the mass dependence of the chiral condensate and its susceptibility. However, no comparison to the universal scaling functions of $O(N)$ spin models was attempted in the vicinity of $\beta_{c}$. In order to achieve that we have made additional simulations at more $\beta$-values around the supposed chiral transition point and at additional and smaller $m_{q} a$-values. A second aim of our paper is the study of the Goldstone effects which are connected to the breaking of chiral symmetry. If aQCD behaves as an effective three-dimensional $O(N)$ spin model close to the chiral transition point then one would expect the chiral susceptibility $\chi_{m}$ to diverge below $\beta_{c}$ as $\left(m_{q} a\right)^{-1 / 2}$ for $m_{q} a \rightarrow 0$. Such a mass dependence has in fact been observed in the range $\beta_{d}<\beta<\beta_{c}$ in Ref. [16]. For smaller $T$-values, approaching $T=0$, the theory changes to an effective fourdimensional theory and the expected divergence of $\chi_{m}$ becomes logarithmic in $m_{q} a$. The change in the behaviours of $\langle\bar{\psi} \psi\rangle$ and $\chi_{m}$ with decreasing $T$, also below $\beta_{d}$, is therefore of interest.

\footnotetext{
${ }^{1}$ We have adopted the QCD convention of counting the number of fermion flavours. Because the fermions are in the adjoint representation one Dirac fermion corresponds to two Majorana fermions.
} 
The paper is organized as follows. In the next section we define the action and the observables of aQCD, which we want to consider. Then we discuss numerical details of our simulations and give an overview of the available data. In Section 3 we recapitulate those results from the $O(N)$ spin models which are most relevant for the subsequent analysis of the aQCD data in Section 4. We close with a summary and the conclusions.

\section{QCD with adjoint fermions on the lattice}

In the following we shall use previous results obtained by Karsch and Lütgemeier and extend their simulations for our purposes. The version of aQCD which we use is therefore the same as the one already described in detail in Ref. [16] and it will suffice to address only the main aspects of the corresponding lattice theory. The action is similar to QCD with staggered fermions in the fundamental representation. The gluon part $S_{G}$ of the action is the standard Wilson one-plaquette gauge action with $S U(3)$ link variables $U^{(3)}$ and coupling $\beta=6 / g^{2}$

$$
S_{G}=\beta \sum_{P}\left\{1-\frac{1}{3} \operatorname{Re} \operatorname{tr} U_{P}^{(3)}\right\} .
$$

In contrast to QCD the fermions are in the eight-dimensional adjoint representation of the $S U(3)$ group and have eight colour degrees of freedom. The fermionic part $S_{F}$ of the action requires then also an eight-dimensional representation $U^{(8)}$ of the link matrices which may be expressed in terms of the three-dimensional matrices

$$
U_{a b}^{(8)}=\frac{1}{2} \operatorname{tr}\left[\lambda_{a} U^{(3)} \lambda_{b} U^{(3) \dagger}\right],
$$

where the $\lambda_{a}$ are the eight Gell-Mann matrices and tr is as in (1) the 3-trace. The total aQCD action is

$$
S=S_{G}+S_{F}=S_{G}+\sum_{x, y} \bar{\psi}_{x} M\left(U^{(8)}\right)_{x, y} \psi_{y} .
$$

Here, $M\left(U^{(8)}\right)$ is the standard staggered fermion matrix where the link variables $U^{(3)}$ have been replaced by the respective $U^{(8)}$ matrices. We note some important properties of $U^{(8)}$. From Eq. (2) it follows that $U^{(8)}$ is real and its 8-trace $\left(\operatorname{tr}_{8}\right)$ is related to the 3 -trace of $U^{(3)}$ via

$$
\operatorname{tr}_{8} U^{(8)}=\left|\operatorname{tr} U^{(3)}\right|^{2}-1 .
$$

Further, $U^{(8)}$ is invariant under $Z(3)$ transformations of the $U^{(3)}$-matrices because the centre elements appear in Eq. (2) as a complex conjugate pair. As a consequence, $S_{F}$ does not break the $Z(3)$ centre symmetry and the Polyakov loop

$$
L_{3}=\left\langle\frac{1}{N_{\sigma}^{3}}\left|\sum_{\vec{x}} \operatorname{tr} \prod_{x_{4}=1}^{N_{\tau}} U^{(3)}\left(x_{4}, \vec{x}\right)\right|\right\rangle,
$$


is an order parameter for the deconfinement transition like in pure $S U(3)$ gauge theory in the limit $N_{\sigma} \rightarrow \infty$. The order parameter for the chiral transition, the chiral condensate, is defined by

$$
\langle\bar{\psi} \psi\rangle=\frac{T}{V} \frac{\partial \ln Z}{\partial m_{q} a}=\frac{1}{N_{\sigma}^{3} N_{\tau}} \frac{N_{f}}{2}\left\langle\operatorname{Tr} M^{-1}\right\rangle
$$

where the trace $\operatorname{Tr}$ has to be taken over the eight colours and all spacepoints. The derivative of the chiral condensate with respect to $m_{q} a$ is the chiral susceptibility

$$
\chi_{m}=\frac{\partial\langle\bar{\psi} \psi\rangle}{\partial m_{q} a}=\frac{T}{V} \frac{\partial^{2} \ln Z}{\partial\left(m_{q} a\right)^{2}} .
$$

It is the sum of the connected term $\chi_{\text {con }}$ and the disconnected term $\chi_{\text {dis }}$. The latter measures the fluctuations of the chiral condensate. The two parts are given by

$$
\begin{aligned}
\chi_{d i s} & =\frac{1}{N_{\sigma}^{3} N_{\tau}}\left(\frac{N_{f}}{2}\right)^{2}\left(\left\langle\left(\operatorname{Tr} M^{-1}\right)^{2}\right\rangle-\left\langle\operatorname{Tr} M^{-1}\right\rangle^{2}\right) \\
\chi_{c o n} & =-\frac{N_{f}}{2} \sum_{x}\left\langle M_{x, 0}^{-1} M_{0, x}^{-1}\right\rangle .
\end{aligned}
$$

In our subsequent analysis we shall utilise the data for the Polykov loop, the chiral condensate and the disconnected part of the susceptibility.

Since the matrices $U^{(8)}$ are real this is also the case for the total fermion matrix $M\left(U^{(8)}\right)$. The determinant of $M\left(U^{(8)}\right)$ may then be represented as a Gaussian integral over real bosonic fields with the advantage that the exact Hybrid Monte Carlo algorithm can be used for the simulation of aQCD with two adjoint fermion flavours. The corresponding effective pseudo-fermion action is given by

$$
S_{e f f}=S_{G}\left(U^{(3)}\right)+\Phi^{t}\left(M\left(U^{(8)}\right)^{t} M\left(U^{(8)}\right)\right) \Phi,
$$

with real eight-component $\Phi$-fields, which reside only on even lattice sites to compensate for the fermion doubling introduced by the use of $M^{t} M$ instead of $M$. The molecular dynamics equations for aQCD are more complicated as the ones for QCD, because $M$ is now quadratic in $U^{(3)}$. Details of the update algorithm can be found in appendix A of Ref. [20].

Our simulations were performed on $N_{\sigma}^{3} \times 4$ lattices with $N_{\sigma}=8,12$ and 16 and a fixed length $\tau=n \delta \tau=0.25$ of the trajectories. The number of molecular dynamics steps $n$ was chosen such that the final Metropolis acceptance rate was in the range of $60-80 \%$. On the $8^{3} \times 4$ lattice $n$ varied between 20 and 40 for $0.10 \geq m_{q} a \geq 0.02$. For lower masses and/or increasing spatial extension $N_{\sigma} a$ of the lattice the number of steps had to be increased. The optimal $n$-value was however relatively independent of $\beta$, apart from the region around and below the deconfinement transition, where larger $n$ were necessary. The chiral condensate and the Polyakov loop were measured 


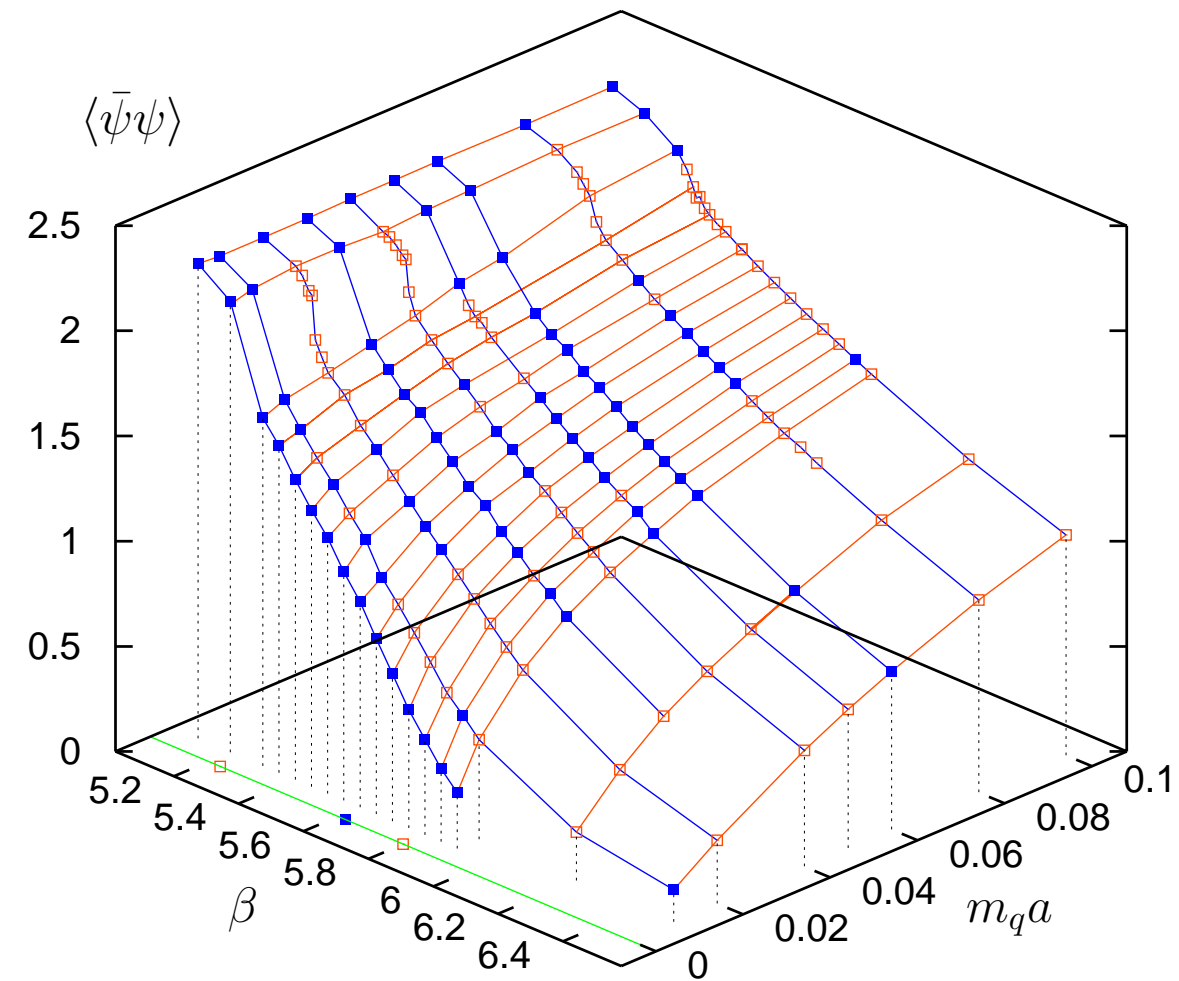

(a)

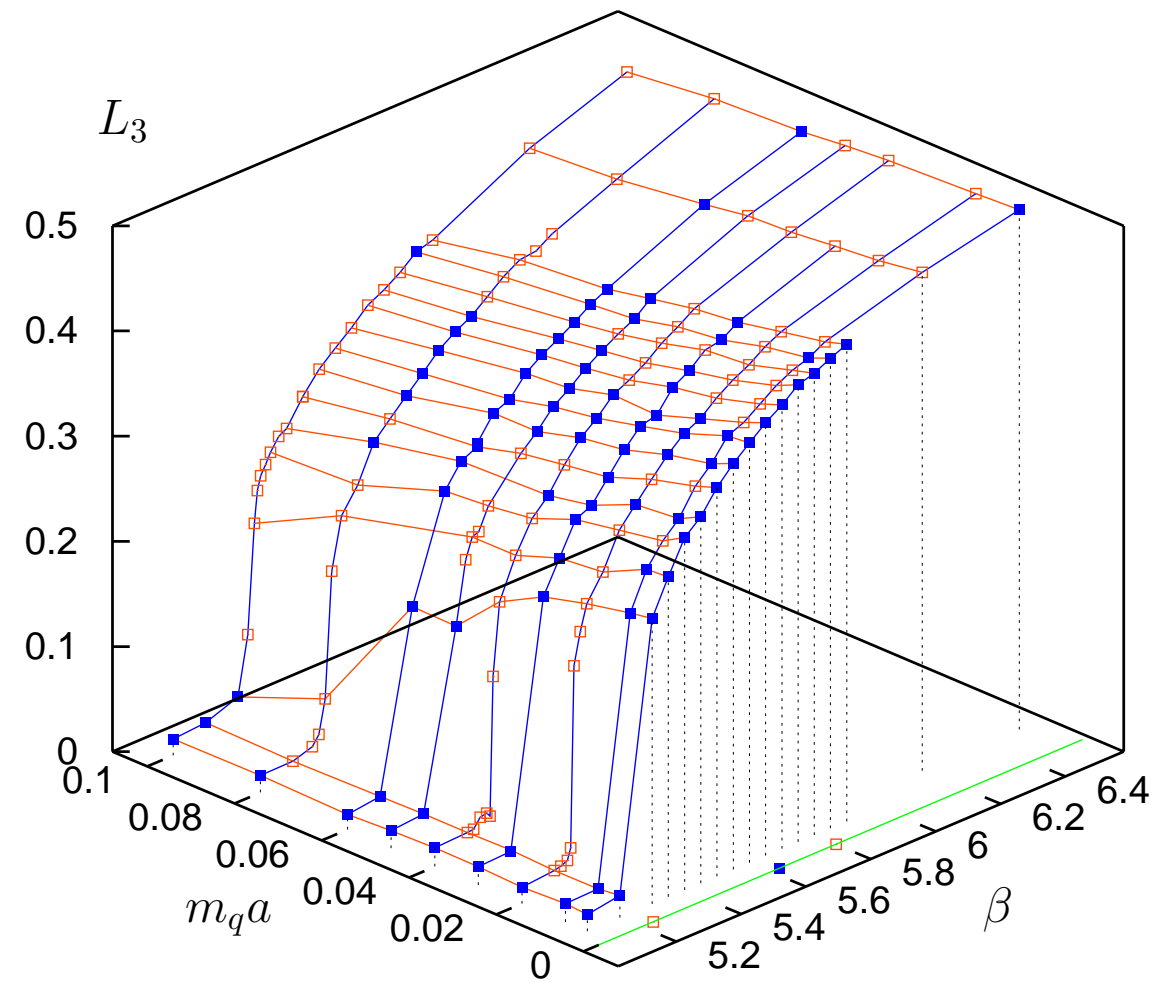

(b)

Figure 1: The chiral condensate $\langle\bar{\psi} \psi\rangle$ (a) and the Polyakov loop $L_{3}$ (b) versus $\beta$ and $m_{q} a$ on the $8^{3} \times 4$ lattice (plot (b) has been rotated by $90^{\circ}$ ). The filled squares denote the new data, the empty ones are from Ref. [16]. The dotted lines indicate the height. The data points are connected by straight lines to guide the eye. 
after the completion of each trajectory. Both the condensate and the disconnected part of the susceptibility were calculated from a noisy estimator [21] with 25 random source vectors. As already mentioned in the introduction, our first intention was to enlarge the data set of Ref. [16]. To this end we have performed additional simulations on the $8^{3} \times 4$ lattice with 900-2000 trajectories for measurements at $m_{q} a=0.005,0.03$ and 0.06 , and for the new $\beta$-values 5.1, 5.45, 5.55, 5.6 and 5.65. The already existing data at $m_{q} a=0.01,0.02,0.04,0.05,0.08$ and 0.10 and at the $\beta$-values 5.2, 5.3, 5.35, 5.4, 5.5, 5.7, 5.75, 5.85, 5.9, 6.2 and 6.5 have been completed to obtain a mesh covering the region from above the chiral phase transition to a point just below the deconfinement transition. In order to see possible finite size dependencies we have carried out further simulations at selected points on larger lattices with $N_{\sigma}=12$ and 16 . The respective data will be show in the figures of Section 4. The chiral condensate data from the different lattices coincide inside the error bars at essentially all points. In the chiral susceptibility data there are however some differences which may be due to insufficient statistics.

An overview of the data from the $8^{3} \times 4$ lattice is given in Fig. 1 where we show three-dimensional plots of the chiral condensate $\langle\bar{\psi} \psi\rangle$ and the Polyakov loop $L_{3}$. In the plots we have indicated on the line at $m_{q} a=0$ the previously [16] determined transition points $\beta_{d}=5.236(3)$ and $\beta_{c}=5.79(5)$ by empty squares. The filled square on the same line denotes our new estimate for $\beta_{c}$, which we will explain in Section 4. Obviously, the chiral phase transition point cannot be determined by a simple inspection of the order parameter $\langle\bar{\psi} \psi\rangle$, even though we have many new data points in the critical region. For the first order deconfinement transition the situation is much clearer. The Polyakov loop $L_{3}$ shows jumps between $\beta=5.2$ and 5.3 , depending on the mass value. Our new results at $\beta=5.1$ and 5.2 are definitely below the deconfinement transition. Insofar they support the determination of the exact position by Karsch and Lütgemeier who produced more data in the transition region at the masses $m_{q} a=0.02,0.04,0.08$ and 0.10 . The straight line connections in Fig. 1 in this region at the other mass values should therefore be taken with care. Two further comments are appropriate here. Already in Ref. [20] a remarkable fact was noted: the first order deconfinement transition point $\beta_{d}=5.236(3)$ of aQCD coincides with the second order chiral transition point $\beta_{c}=5.233(7)$ of QCD with two fundamental quarks and the standard staggered action [9]. In addition, we see in Fig. 1 that $\langle\bar{\psi} \psi\rangle$ exhibits also a discontinuity at $\beta_{d}$, whereas the Polyakov loop shows no sign of the chiral transition.

\section{The three-dimensional $O(N)$ spin models}

In this section we want to summarize the basic facts about the three-dimensional $O(N)$ spin models and their universality classes. A more general survey on $O(N)$ symmetric systems can be found in Ref. [22]. The numerical results to which we will 
refer in the following have been obtained from simulations of the standard $O(N)$ invariant non-linear sigma models with the partition function

$$
Z=\int[d \vec{\phi}] \exp \left[J \sum_{x, y} \vec{\phi}_{x} \cdot \vec{\phi}_{y}+\vec{H} \cdot \sum_{x} \vec{\phi}_{x}\right]
$$

Here, $x$ and $y$ are nearest-neighbour sites on a three-dimensional hypercubic lattice and $\vec{\phi}_{x}$ is an $N$-component unit vector at site $x$. The coupling $J$ acts as inverse temperature $J=1 / T$ and $\vec{H}$ is the magnetic field. The spin vector $\vec{\phi}_{x}$ may be decomposed into a longitudinal (parallel to $\vec{H}$ ) and a transverse component (perpendicular to $\vec{H}): \vec{\phi}_{x}=\phi_{x}^{\|} \hat{\vec{H}}+\vec{\phi}_{x}^{\perp}$. The order parameter of the system, the magnetization $M$ and its susceptibility $\chi_{L}$ are then defined by

$$
M=\left\langle\frac{1}{V} \sum_{x} \phi_{x}^{\|}\right\rangle=\left\langle\phi^{\|}\right\rangle, \quad \chi_{L}=\frac{\partial M}{\partial H}=V\left(\left\langle\phi^{\| 2}\right\rangle-M^{2}\right)
$$

that is, $M$ is the expectation value of the lattice average of the longitudinal spin component and $\chi_{L}$ its derivative with respect to $H=|\vec{H}|$ (implying $H \geq 0$ ).

The spin system exhibits a second order phase transition at a critical temperature $T_{c}=1 / J_{c}$. For $H \rightarrow 0$ and $T<T_{c}$ the $O(N)$-symmetry of the spin system is spontaneously broken and the magnetization attains a finite value $M(T, 0)>0$, whereas $M\left(T>T_{c}, 0\right)=0$. The behaviour of $M(T, H)$ in the region around the critical point is described by the equation of state

$$
M=h^{1 / \delta} f_{G}(z), \quad \text { with } \quad z=t / h^{1 / \beta \delta}
$$

where $f_{G}(z)$ is a universal scaling function, which is normalized such that

$$
f_{G}(0)=1, \text { and } \quad f_{G}(z) \underset{z \rightarrow-\infty}{\longrightarrow}(-z)^{\beta} .
$$

This is achieved by the introduction of the reduced temperature $t$ and field $h$ with

$$
t=\left(T-T_{c}\right) / T_{0} \quad \text { and } \quad h=H / H_{0}
$$

such that

$$
M(t=0, h)=h^{1 / \delta}, \quad M(t<0, H \rightarrow 0)=(-t)^{\beta} .
$$

The critical exponents $\delta$ and $\beta$ determine all other critical exponents via the hyperscaling relations (here $d=3$ )

$$
\gamma=\beta(\delta-1), \quad d \nu=\beta(1+\delta)=2-\alpha, \quad 2-\eta=\gamma / \nu
$$

The scaling function $f_{G}(z)$ and the critical exponents are the same for all members of the corresponding universality class. They can therefore be used in tests on the class of a second order phase transition. In a series of papers these functions have been calculated for the three-dimensional $O(N)$ models with $N=2[23], N=4$ 


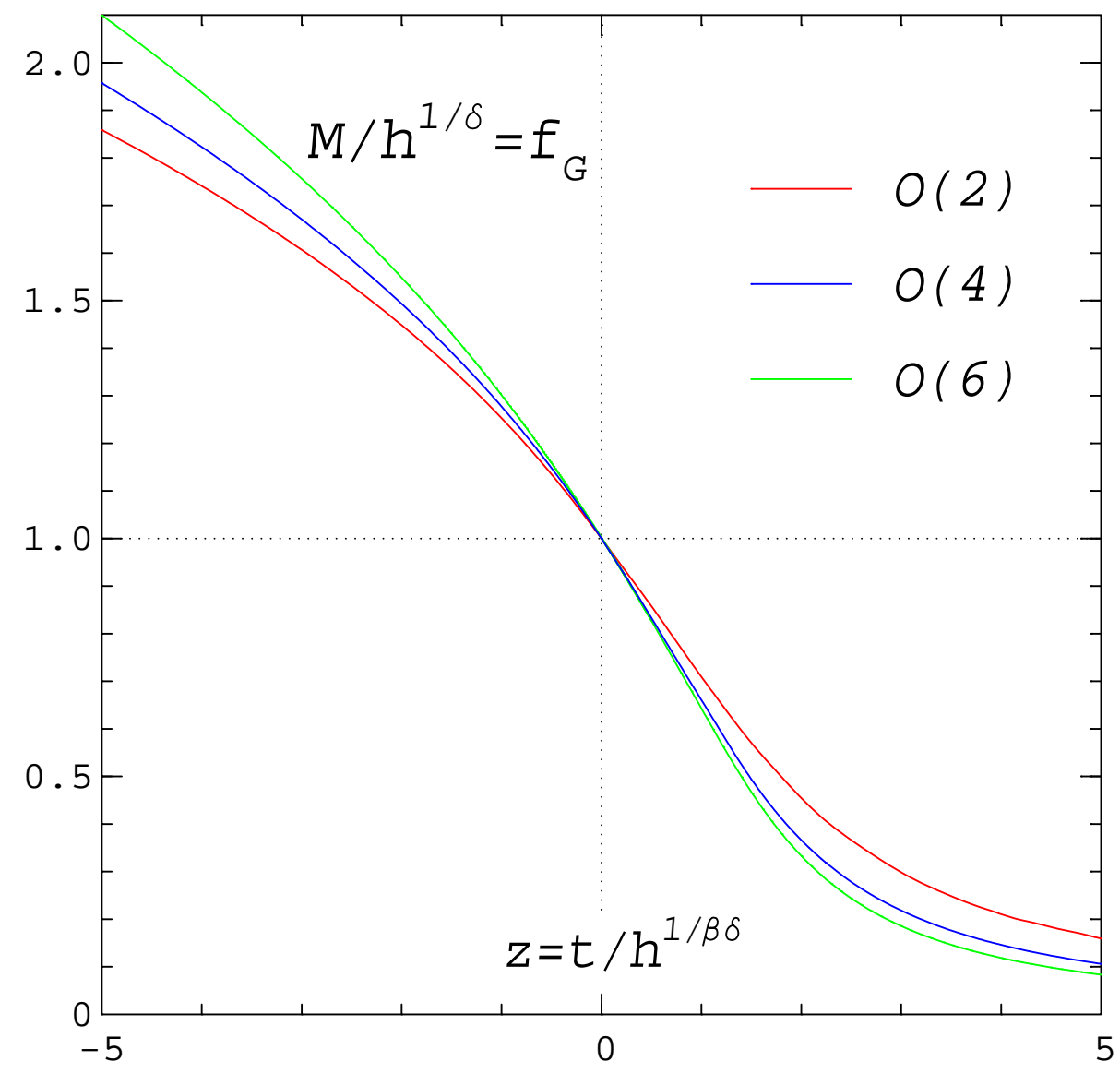

Figure 2: The universal scaling function $f_{G}=M h^{-1 / \delta}$ versus the scaling variable $z=t h^{-1 / \beta \delta}$ for $N=2$ [23], $N=4$ [26] and $N=6$ [27]. Parametrizations of the curves can be found in Refs. [23, 26, 27].

$[24,25,26]$ and $N=6[27]$. They are shown in Fig. 2. We observe similar shapes of the different $f_{G}(z)$-curves with an increasing slope for larger $N$. In the plot the critical line $T=T_{c}$ is mapped to the point $z=0$, the symmetric (high temperature) phase $T>T_{c}, H \rightarrow 0$ corresponds to the limit $z \rightarrow \infty$ and the coexistence line $T<T_{c}, H \rightarrow 0$ to $z \rightarrow-\infty$. Since the exponents $\delta$ and $\beta$ are slightly different for each $N$ the scales of both axes of the plot vary with $N$. In Table 1 we have listed the critical exponents which have been used in Fig. 2. We see that the exponent $\beta$

\begin{tabular}{|c||c|c|c|c||c|c||c|}
\hline$N$ & $\beta$ & $\delta$ & $\nu$ & $\alpha$ & $1 / \delta$ & $1 / \beta \delta$ & Ref. \\
\hline 2 & 0.349 & 4.7798 & 0.6724 & -0.017 & 0.2092 & 0.5995 & {$[23]$} \\
4 & 0.380 & 4.824 & 0.7377 & -0.213 & 0.2073 & 0.5455 & {$[26]$} \\
6 & 0.425 & 4.780 & 0.818 & -0.454 & 0.2092 & 0.4922 & {$[27]$} \\
\hline
\end{tabular}

Table 1: The critical exponents of the $3 d O(N)$ spin models. 
is increasing with $N$, while $\delta$ is nearly constant. The main influence of $N$ in a scaling plot is therefore coming from the $N$-dependence of the product $\Delta=\beta \delta$ which appears in the scaling variable $z$.

Since the susceptibility $\chi_{L}$ is the derivative of $M$ with respect to $H$, it can as well be expressed in terms of a universal scaling function

$$
\chi_{L}=\frac{\partial M}{\partial H}=\frac{h^{1 / \delta-1}}{H_{0}} f_{\chi}(z)
$$

with

$$
f_{\chi}(z)=\frac{1}{\delta}\left(f_{G}(z)-\frac{z}{\beta} f_{G}^{\prime}(z)\right) .
$$

The scaling function $f_{\chi}(z)$ has a peak at a positive value $z=z_{p c}$. Correspondingly, $\chi_{L}$ has a maximum for each fixed value $h$ at a temperature $t_{p c}(h)$. This function of $h$ defines the pseudocritical line in the $(t, h)$-plane for $t \geq 0$. Obviously, it is given by

$$
t_{p c} h^{-1 / \Delta}=z_{p c} \quad \text { or } \quad T_{p c}-T_{c}=c H^{1 / \Delta}
$$

where $c=z_{p c} T_{0} H_{0}^{-1 / \Delta}$ is a non-universal constant. Eq. (20) is often used to determine $T_{c}$.

Due to the existence of massless Goldstone modes in $O(N)$ models with $N>1$ and dimension $2<d \leq 4$ singularities are expected on the whole coexistence line $T<T_{c}, H=0[28,29,30]$ in addition to the known critical behaviour at $T_{c}$. In particular, the susceptibility $\chi_{L}$ is diverging on the coexistence line. For all $T<T_{c}$ and $H \rightarrow 0$ the divergence is

$$
\chi_{L}(T, H) \sim\left\{\begin{array}{ll}
H^{-1 / 2} & \text { for } d=3 \\
\ln H & \text { for } d=4
\end{array},\right.
$$

which implies the following $H$-dependence at fixed $T$ for the magnetization

$$
M(T, H)=M(T, 0)+c_{1}(T) \cdot\left\{\begin{array}{ll}
H^{1 / 2} & \text { for } d=3 \\
H(\ln H-1) & \text { for } d=4
\end{array} .\right.
$$

For the three-dimensional $O(N)$ models with $N=2,4,6$ the $H^{1 / 2}$-dependence of the magnetization for $T<T_{c}$ has been verified numerically in Refs. [23, 25, 27]. In all cases one finds that the slope $c_{1}(T)$ is small for very low temperatures, that is $M$ is essentially constant. Approaching $T_{c}$, the slope increases and the region close to $H=0$ where $M$ is a straight line as a function of $H^{1 / 2}$ shrinks. This is of course what one expects, because at $T_{c}$ the magnetization changes its $H$-dependence from $H^{1 / 2}$ to $H^{1 / \delta}$ with the effect that $c_{1} \rightarrow \infty$. The scaling functions $f_{G}$ and $f_{\chi}$ describe this behaviour correctly in the critical region. Yet, Eqs. (21) and (22) are also valid for all temperatures below the critical region. 


\section{Analysis of the aQCD data}

In aQCD the temperature is given by $T=1 /\left(N_{\tau} a\right)$ and the analogon of the magnetic field is the quark mass $m_{q} a$. Since the lattice spacing $a$ is a monotone function of the coupling $\beta$, we can use $\beta$ instead of the temperature and temperature differences are proportional to coupling differences. Accordingly, we define instead of the reduced temperature $t$ a reduced coupling $\beta_{r}$ and a reduced field $m$, where

$$
\beta_{r}=\left(\beta-\beta_{c}\right) / \beta_{0}, m=\left(m_{q} a\right) /\left(m_{q} a\right)_{0} \quad \text { and } \quad z=\beta_{r} m^{-1 / \Delta} .
$$

The constants $\beta_{0}$ and $\left(m_{q} a\right)_{0}$ have to be fixed from the normalization of the scaling function for $\langle\bar{\psi} \psi\rangle$. It is clear, that before any scaling tests can be carried out, the critical point $\beta_{c}$ of the chiral transition has to be determined as accurately as possible.

\subsection{Location of the chiral transition point}

We start our search by following first the usual strategy to extrapolate the pseudocritical couplings $\beta_{p c}\left(m_{q} a\right)$ to $m_{q} a=0$ with an ansatz equivalent to Eq. (20)

$$
\beta_{p c}=\beta_{c}+c\left(m_{q} a\right)^{1 / \Delta}
$$

The $\beta_{p c}$ are in principle the positions of the maxima of $\chi_{m}$. In Ref. [7] the two parts $\chi_{d i s}$ and $\chi_{\text {con }}$ of $\chi_{m}$ have been studied seperately. It was found that the contribution of the connected part to $\chi_{m}$ is a slowly varying function of $\beta$ in the critical region and that the ratio $\chi_{\text {con }} / \chi_{\text {dis }}$ decreases with decreasing quark mass. It suffices therefore to take the peak positions of $\chi_{d i s}$ for the extrapolation to the critical point. The data for the susceptibility $\chi_{\text {dis }}$ from the $8^{3} \times 4$ lattice are shown in Fig. 3 for the three smallest masses $0.005,0.01$ and 0.02 . They have been interpolated by reweighting where we had enough statistics. We observe a pronounced but broad maximum for the smallest mass 0.005 around $\beta \approx 5.73$, for $m_{q} a=0.01$ a less peaked and smaller maximum around $\beta \approx 5.74$ and for $m_{q} a=0.02$ only a long and low plateau between $\beta=5.65$ and $\beta=5.85$. In Table 2 we have listed our estimates for the pseudocritical couplings. For $m_{q} a=0.01$ and 0.02 they differ somewhat from the values obtained in Ref. [16] because we have more data points, the value at $m_{q} a=0.005$ is new. Certainly, the errors on $\beta_{p c}\left(m_{q} a\right)$ are too large to lead to a precise value of the

\begin{tabular}{|c||c|c|c|}
\hline$m_{q} a$ & 0.005 & 0.010 & 0.020 \\
\hline$\beta_{p c}$ & $5.73(8)$ & $5.74(6)$ & $5.75(10)$ \\
\hline
\end{tabular}

Table 2: The pseudocritical couplings $\beta_{p c}$ from the $8^{3} \times 4$ lattice. 


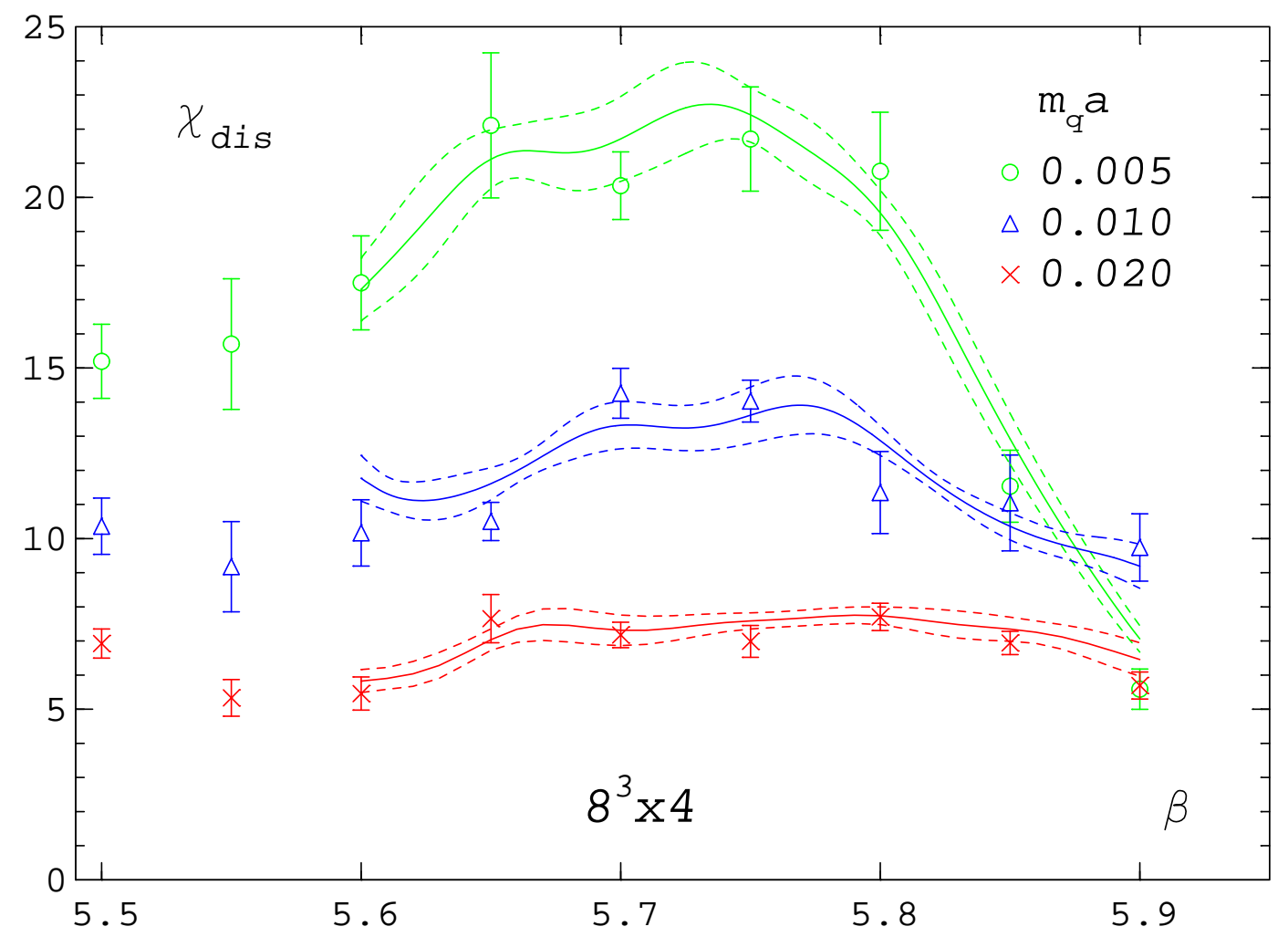

Figure 3: The susceptibility $\chi_{d i s}$ as a function of $\beta$ for the three smallest masses $m_{q} a=0.005,0.01$ and 0.02 on the $8^{3} \times 4$ lattice. The solid lines are the results from reweighting, the dashed lines show the Jackknife error corridor.

critical point $\beta_{c}$. On the other hand it is clear that $\beta_{c}$ must be definitely smaller than the $\beta_{p c}\left(m_{q} a\right)$ because the susceptibility peaks only in the high temperature phase $\beta_{r}>0$. If one nevertheless tries an extrapolation with Eq. (20) using the numbers from Table 2 and $O(2)$ and $O(6)$ values for $\Delta$ one obtains $\beta_{c}=5.72(16)$ and 5.71(19), respectively.

A second possibility to locate the critical point is based on the expected mass dependence of the order parameter at $\beta=\beta_{c}$

$$
\langle\bar{\psi} \psi\rangle=d_{c}\left(m_{q} a\right)^{1 / \delta} .
$$

The method consists in a comparison of the mass dependence of the data at each $\beta$-value with this functional form. In practice one makes straight line fits at fixed $\beta$ of the logarithm of the order parameter as a function of $\ln \left(m_{q} a\right)$

$$
\ln \langle\bar{\psi} \psi\rangle=\ln d_{c}+\frac{1}{\delta} \ln \left(m_{q} a\right) .
$$

In the vicinity of $\beta_{c}$ one should find the lowest $\chi^{2} /$ d.o.f. and/or the largest fit range in $m_{q} a$. In addition, one obtains a first result for $1 / \delta$. A method of this type was successfully applied to the corresponding finite size scaling problem in $S U(2)$ gauge 


\begin{tabular}{|c||c|c|c|c|c||c|}
\hline$\beta$ & $\ln d_{c}$ & $1 / \delta$ & $m_{q} a$-range & $N_{p}$ & $\chi_{\min }^{2} /$ d.o.f. & $\chi_{\max }^{2} /$ d.o.f. \\
\hline 5.80 & $1.510(37)$ & $0.4246(97)$ & $0.005-0.03$ & 4 & 7.51 & 32.63 \\
\hline 5.75 & $1.336(25)$ & $0.3549(65)$ & $0.005-0.03$ & 4 & 4.44 & 28.00 \\
\hline 5.70 & $1.124(23)$ & $0.2786(60)$ & $0.005-0.03$ & 4 & 1.05 & 10.43 \\
\hline 5.65 & $1.026(09)$ & $0.2322(26)$ & $0.005-0.06$ & 7 & 1.64 & 3.00 \\
\hline 5.60 & $0.949(04)$ & $0.1921(17)$ & $0.005-0.10$ & 9 & 5.25 & 9.11 \\
\hline 5.55 & $0.822(19)$ & $0.1423(50)$ & $0.005-0.04$ & 5 & 4.18 & 7.51 \\
\hline 5.50 & $0.755(19)$ & $0.1083(46)$ & $0.005-0.03$ & 4 & 5.62 & 8.79 \\
\hline
\end{tabular}

Table 3: The parameters of the fits according to Eq. (26). The number $N_{p}$ of fitted data points and the fit range correspond to the minimal $\chi^{2} /$ d.o.f. with $N_{p} \geq 4$. The last column is the maximal $\chi^{2} /$ d.o.f., if $N_{p}$ is varied between 4 and 9 .

theory [31]. In Table 3 we present the results for all fits in the interval $5.5 \leq \beta \leq 5.8$. At each $\beta$-value we have nine data points in the range $0.005 \leq m_{q} a \leq 0.10$. Because we have two fit parameters we used at least the data points from the four smallest masses, if possible more, up to all the nine points. The best $\chi^{2} / d$.o.f. is obtained at $\beta=5.7$ and 5.65 , but for $\beta=5.7$ only in the smallest mass interval. At $\beta=5.65$ there is only a small variation in $\chi^{2} /$ d.o.f. when $N_{p}$ is increased. For $\beta=5.60$ we find a larger $\chi^{2} /$ d.o.f., which is due to the lowest mass point, but the best fit includes all nine data points. The data points at $m_{q} a=0.005,0.01$ and 0.02 from the $8^{3} \times 4$ lattice agree here within the errorbars with those from the $16^{3} \times 4$ lattice, so that a finite size effect is improbable. In conclusion, the range $5.6 \leq \beta \leq 5.7$ contains presumably the critical point $\beta_{c}$. If we look at the corresponding $1 / \delta$-values from the fits we find $0.1921 \leq 1 / \delta \leq 0.2786$. That interval includes the value 0.2092 , which is expected from the $O(2)$ and $O(6)$ spin models, suggesting $5.6 \leq \beta_{c} \leq 5.65$.

We can further improve the search for the critical point by assuming a scaling ansatz for the order parameter

$$
\langle\bar{\psi} \psi\rangle=m^{1 / \delta} f(z)
$$

as in spin models. Expanding $f(z)$ for small $|z|$ one obtains

$$
\begin{aligned}
\langle\bar{\psi} \psi\rangle & =m^{1 / \delta}\left(f(0)+z f^{\prime}(0)+\ldots\right) \\
& =\left(m_{q} a\right)^{1 / \delta}\left(d_{c}+d_{c}^{1}\left(\beta-\beta_{c}\right)\left(m_{q} a\right)^{-1 / \Delta}+\ldots\right) .
\end{aligned}
$$

Here we have used the normalization condition $f(0)=1$ and Eqs. (23) and (25). They imply $d_{c}=\left(m_{q} a\right)_{0}^{-1 / \delta}$ and a similar relation for the amplitude $d_{c}^{1}$. The advantage of Eq. (29) is, that it is valid also in the vicinity of $\beta_{c}$ and at medium size values of $m_{q} a$. The mass should not be too small to keep $|z|$ small and not too large to be inside the critical region where (27) is applicable. We have made fits 


\begin{tabular}{|c||c|c|c|c|}
\hline$\beta$ & $d_{c}$ & $d_{c}^{1}\left(\beta-\beta_{c}\right)$ & $m_{q} a$-range & $\chi^{2} /$ d.o.f. \\
\hline 5.90 & $2.736(8)$ & $-0.1101(16)$ & $0.03-0.10$ & 2.19 \\
\hline 5.85 & $2.704(8)$ & $-0.0868(14)$ & $0.02-0.10$ & 1.53 \\
\hline 5.80 & $2.706(6)$ & $-0.0713(12)$ & $0.02-0.10$ & 1.16 \\
\hline 5.75 & $2.689(6)$ & $-0.0508(11)$ & $0.02-0.10$ & 1.30 \\
\hline 5.70 & $2.662(6)$ & $-0.0283(10)$ & $0.01-0.10$ & 1.56 \\
\hline 5.65 & $2.654(5)$ & $-0.0089(08)$ & $0.005-0.10$ & 1.68 \\
\hline 5.60 & $2.659(7)$ & $0.0080(13)$ & $0.01-0.10$ & 2.97 \\
\hline 5.55 & $2.624(9)$ & $0.0342(20)$ & $0.03-0.10$ & 2.30 \\
\hline 5.50 & $2.606(13)$ & $0.0583(24)$ & $0.03-0.10$ & 1.99 \\
\hline
\end{tabular}

Table 4: The parameters for the fit (29) with $O(2)$ exponents.

\begin{tabular}{|c||c|c|c|c|}
\hline$\beta$ & $d_{c}$ & $d_{c}^{1}\left(\beta-\beta_{c}\right)$ & $m_{q} a$-range & $\chi^{2} /$ d.o.f. \\
\hline 5.90 & $2.862(8)$ & $-0.1722(16)$ & $0.02-0.10$ & 2.43 \\
\hline 5.85 & $2.811(9)$ & $-0.1449(21)$ & $0.01-0.10$ & 2.89 \\
\hline 5.80 & $2.793(6)$ & $-0.1192(17)$ & $0.01-0.10$ & 0.75 \\
\hline 5.75 & $2.748(6)$ & $-0.0841(15)$ & $0.01-0.10$ & 1.20 \\
\hline 5.70 & $2.693(6)$ & $-0.0463(14)$ & $0.005-0.10$ & 2.39 \\
\hline 5.65 & $2.669(6)$ & $-0.0159(15)$ & $0.005-0.10$ & 1.73 \\
\hline 5.60 & $2.647(8)$ & $0.0141(22)$ & $0.01-0.10$ & 2.70 \\
\hline 5.55 & $2.585(11)$ & $0.0563(34)$ & $0.03-0.10$ & 2.07 \\
\hline 5.50 & $2.538(15)$ & $0.0961(40)$ & $0.03-0.10$ & 1.48 \\
\hline
\end{tabular}

Table 5: The parameters for the fit (29) with $O(6)$ exponents.

with Eq. (29) and $O(2)$ and $O(6)$ exponents alternatively. The results are given in Tables 4 and 5 . The shown fit range in $m_{q} a$ extends always to $m_{q} a=0.10$. The lower limit of the range was chosen such that the next smaller mass would increase $\chi^{2} /$ d.o.f. considerably. We observe that the ranges extend to the smallest masses for $5.6 \leq \beta \leq 5.7$. The result for the amplitude $d_{c}$ should of course be independent of the used $\beta$-value as long as $\beta$ is in the critical region. For $O(2)$ exponents (Table 4) the amplitude result is generally less varying than for $O(6)$ exponents (Table 5), in the range $5.6 \leq \beta \leq 5.7$ the result for $O(2)$ is actually constant within error bars. If we consider this $\beta$-range as the effective critical region we obtain the value $d_{c}=2.66(1)$ for $O(2)$ and $d_{c}=2.67(3)$ for $O(6)$ exponents. The assessment of the range $5.6 \leq \beta \leq 5.7$ as the critical region is further supported by the parameter $d_{c}^{1}\left(\beta-\beta_{c}\right)$, because it has a zero in this region. In Fig. 4 we have plotted the results 


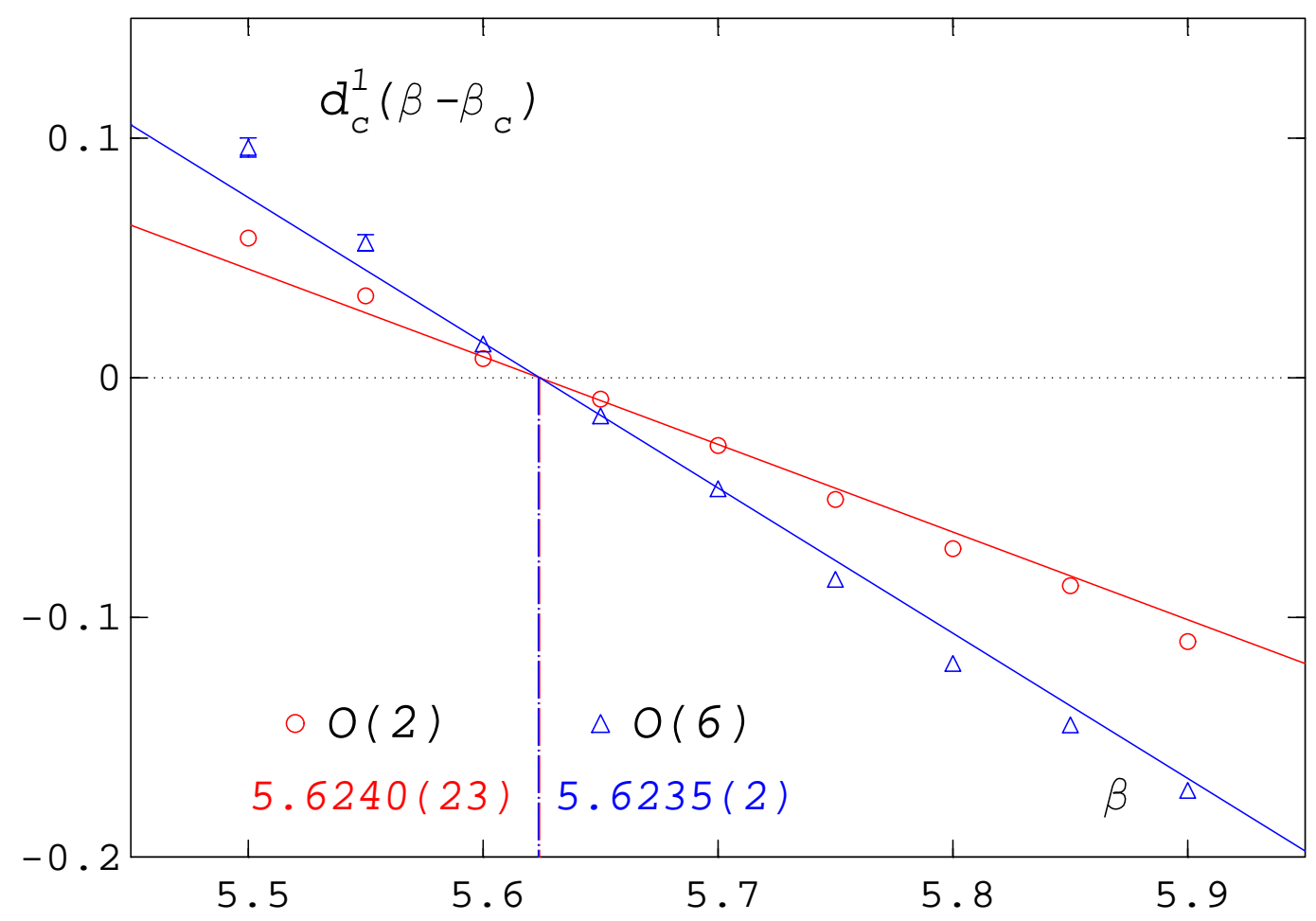

Figure 4: The parameter $d_{c}^{1}\left(\beta-\beta_{c}\right)$ as a function of $\beta$ for $O(2)$ (circles) and $O(6)$ exponents (triangles). The straight lines are fits to the values at $\beta=5.6,5.65$ and 5.7. The zeros of the fits are given by the numbers in the plot.

for this parameter and straight line fits to the three values in the critical region. We obtain coinciding values for $\beta_{c}: 5.6240(23)$ for the $O(2)$ and $5.6235(2)$ for the $O(6)$ case. The errors on these results should however not be overestimated, because they are only those from the straight line fits. Nonetheless we have arrived at a remarkably clear estimate for $\beta_{c}$. From $\beta_{c}$ and $\beta_{d}$ we can derive the ratio $T_{c} / T_{d}$ of the critical temperatures approximately with the 2-loop $\beta$-function

$$
a \Lambda_{L} \approx R(\beta)=\left(\frac{\beta}{6 b_{0}}\right)^{b_{1} / 2 b_{0}^{2}} \exp \left(-\frac{\beta}{12 b_{0}}\right) .
$$

The coefficients $b_{0}$ and $b_{1}$ for aQCD are given by [32]

$$
b_{0}=\frac{3}{16 \pi^{2}}, \quad b_{1}=-\frac{90}{\left(16 \pi^{2}\right)^{2}},
$$

and the temperature ratio becomes

$$
\frac{T_{c}}{T_{d}}=\frac{a\left(\beta_{d}\right)}{a\left(\beta_{c}\right)} \approx\left(\frac{\beta_{c}}{\beta_{d}}\right)^{5} \exp \left(\frac{4 \pi^{2}}{9}\left(\beta_{c}-\beta_{d}\right)\right)=7.8(2) .
$$

Though we have a lower estimate for $\beta_{c}$ than Ref. [16] we nevertheless arrive at about the same ratio. That is due to the different value for $b_{1}$, which was wrongly taken as $+115 /\left(384 \pi^{4}\right)$ in [16]. 


\subsection{The Goldstone effect}

We have just successfully applied the scaling ansatz (27) to the chiral condensate data. One might therefore directly try to compare the data with the universal scaling functions $f_{G}(z)$ of the spin models. Before we can do so, however, we have to fix the normalization $\beta_{0}$ of the reduced temperature $\beta_{r}$ (the normalization of $m$ is already known from $d_{c}$ ). The amplitude $d_{c}^{1}$ does not fix $\beta_{0}$ because the expression $z d f / d z$ in Eq. (28) is independent of any normalization of $z$ or $\beta_{r}$. For that purpose the critical amplitude of the chiral condensate on the coexistence line has to be determined. In the case of the three-dimensional $O(N)$ spin models the magnetization data for $T<T_{c}$ and $H>0$ have been extrapolated to $H=0$ using Eq. (22). We can proceed in the same manner to obtain $\langle\bar{\psi} \psi\rangle$-values at $m_{q} a=0$, supposed the corresponding Goldstone ansatz

$$
\langle\bar{\psi} \psi\rangle\left(\beta, m_{q} a\right)=\langle\bar{\psi} \psi\rangle(\beta, 0)+c_{1}(\beta)\left(m_{q} a\right)^{1 / 2}+c_{2}(\beta)\left(m_{q} a\right)+\ldots
$$

for $\beta<\beta_{c}$ is meaningful at all. In Fig. 5 we have plotted the chiral condensate data for all $\beta$-values between 5.3 and 5.9 versus $\left(m_{q} a\right)^{1 / 2}$. Here, also the available data from the $12^{3} \times 4$ and $16^{3} \times 4$ lattices are shown. Since there are no perceptible finite

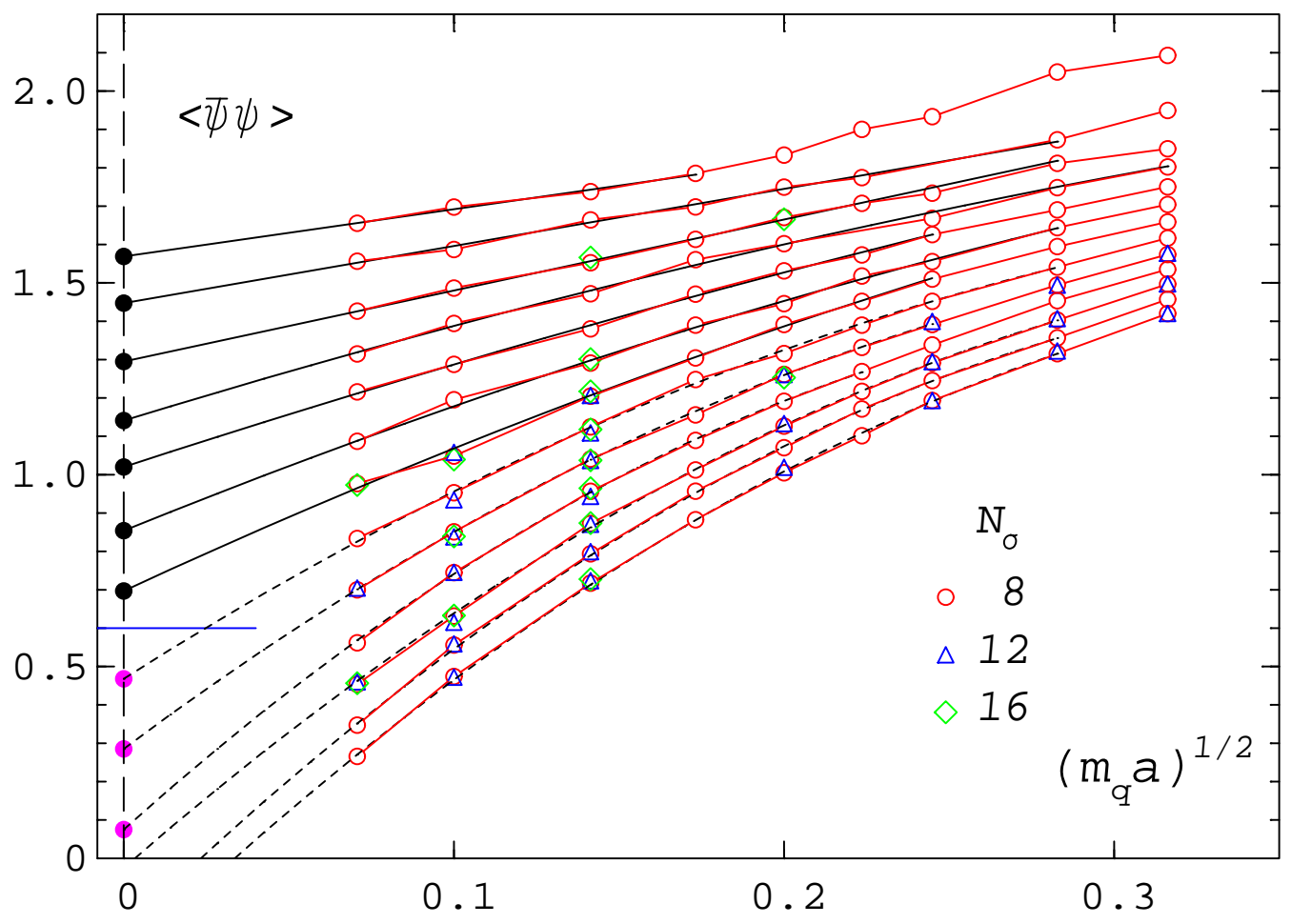

Figure 5: The chiral condensate $\langle\bar{\psi} \psi\rangle$ as a function of $\left(m_{q} a\right)^{1 / 2}$ for all $\beta$-values between 5.3 (highest values) and 5.9 (lowest values) from $N_{\sigma}^{3} \times 4$ lattices with $N_{\sigma}=8$ (circles), 12 (triangles) and 16 (diamonds). The lines are fits with the ansatz (33), the filled circles denote the extrapolations to $m_{q} a=0$. 


\begin{tabular}{|c||c|c|c|c|c|}
\hline$\beta$ & $\langle\bar{\psi} \psi\rangle(\beta, 0)$ & $c_{1}$ & $c_{2}$ & $m_{q} a$-range & $\chi_{\min }^{2} /$ d.o.f. \\
\hline 5.30 & $1.569(13)$ & $1.23(12)$ & - & $0.005-0.03$ & 0.24 \\
\hline 5.35 & $1.447(07)$ & $1.49(04)$ & - & $0.005-0.08$ & 1.33 \\
\hline 5.40 & $1.295(08)$ & $1.85(04)$ & - & $0.005-0.08$ & 0.63 \\
\hline 5.45 & $1.141(11)$ & $2.64(11)$ & $-1.72(25)$ & $0.005-0.10$ & 3.03 \\
\hline 5.50 & $1.020(21)$ & $2.81(26)$ & $-1.37(74)$ & $0.005-0.06$ & 1.19 \\
\hline 5.55 & $0.854(22)$ & $3.49(22)$ & $-2.48(54)$ & $0.005-0.08$ & 2.18 \\
\hline 5.60 & $0.697(24)$ & $3.98(29)$ & $-2.66(84)$ & $0.005-0.06$ & 1.67 \\
\hline 5.65 & $0.468(17)$ & $5.48(20)$ & $-5.97(54)$ & $0.005-0.08$ & 1.33 \\
\hline 5.70 & $0.285(19)$ & $6.44(22)$ & $-7.83(61)$ & $0.005-0.06$ & 1.28 \\
\hline 5.75 & $0.075(22)$ & $7.74(30)$ & $-10.77(96)$ & $0.005-0.05$ & 0.61 \\
\hline 5.80 & $-0.025(19)$ & $7.51(19)$ & $-8.71(45)$ & $0.005-0.08$ & 1.26 \\
\hline 5.85 & $-0.190(12)$ & $8.39(14)$ & $-10.34(37)$ & $0.005-0.08$ & 1.14 \\
\hline 5.90 & $-0.267(10)$ & $8.27(12)$ & $-9.46(31)$ & $0.005-0.08$ & 1.97 \\
\hline
\end{tabular}

Table 6: The parameters of the fits according to the Goldstone ansatz (33). The upper fit boundary was fixed by the minimal $\chi^{2} /$ d.o.f. given in the last column.

size effects, the correlation length must still be small even at the lowest masses. Because we have no data for masses below $m_{q} a=0.005$ we cannot see directly from Fig. 5 at which $\beta$ the Goldstone behaviour, Eq. (33), changes to Eq. (25), the behaviour at the critical point. Nevertheless we have made fits to the data at each $\beta$-value according to Eq. (33), including the first three terms. In Table 6 we present the results of the fits, the corresponding curves are shown in Fig. 5. For $\beta=5.3$, 5.35 and 5.4 the parameter $c_{2}$ is zero within error bars. At $\beta=5.3$ the influence of the nearby deconfinement transition can explicitly be seen at the higher masses $m_{q} a \geq 0.04$, at $\beta=5.35$ for $m_{q} a=0.1$. The fitcurves for $\beta<\beta_{c}$ are shown as solid lines. The short horizontal line in Fig. 5 separates the extrapolated points below and above the critical point. Obviously, the data meet the Goldstone expectations for a three-dimensional theory quite well.

The fitcurves for $\beta>\beta_{c}$ are shown as dashed lines and since they reproduce the data for $m_{q} a \geq 0.005$ as well with a small $\chi^{2} /$ d.o.f., the fits as such provide no clear sign for the location of $\beta_{c}$. However, they provide an upper limit for $\beta_{c}$ insofar as $\langle\bar{\psi} \psi\rangle$ at $m_{q} a=0$ should be positive. From Table 6 we see that this is not the case for $\beta>5.8$. At $\beta \approx 5.8$ the extrapolation is zero and because of that $\beta_{c}$ was located near to 5.8 in Ref. [16]. A hint to the genuine critical point is provided by the $\beta$-dependence of the parameter $c_{2}$. With increasing coupling $c_{2}$ increases slowly, at $\beta=5.65$ however it jumps suddenly to a higher value indicating the change of 
the behaviour from Eq. (33) to Eq. (25). We have also tried fits for $5.3 \leq \beta<\beta_{c}$ with a Goldstone ansatz corresponding to a four-dimensional theory

$$
\langle\bar{\psi} \psi\rangle\left(\beta, m_{q} a\right)=\langle\bar{\psi} \psi\rangle(\beta, 0)+d_{1}(\beta)\left[\left(m_{q} a\right) \ln \left(m_{q} a\right)-\left(m_{q} a\right)\right]+\ldots
$$

The data are not well reproduced with such an ansatz and the respective $\chi^{2} /$ d.o.f. values are about 10-50 times larger than those in Table 6 . That is we confirm that aQCD at finite temperatures in the range $T_{d}<T<T_{c}$ behaves as an effective three-dimensional spin model.

In Fig. 6 we have plotted the disconnected part of the chiral susceptibility $\chi_{\text {dis }}$ for $\beta=5.6$ and 5.65, that is just below and above the critical point $\beta_{c}$. A clear finite size effect is not visible, possibly because our statistics are too low. However, we notice a change in the mass dependence. Below the critical point $\chi_{\text {dis }}$ seems to follow the behaviour expected for $\chi_{m}$ from the Goldstone ansatz (33)

$$
\chi_{m}\left(\beta, m_{q} a\right)=c_{2}+\frac{c_{1}}{2}\left(m_{q} a\right)^{-1 / 2},
$$

whereas above $\beta_{c}$ the increase in the susceptibility as a function of the mass seems to be stronger. A similar change in the mass dependence of $\chi_{\text {dis }}$ from $\beta=5.5$ to 5.7 can be seen in Fig. 4 of Ref. [16]. For comparison we show in Fig. 6 also the predictions for $\chi_{m}$ following from Eq. (35) and the parameters from Table 6 . At $\beta=5.6$ the agreement with the data suggests that here $\chi_{c o n}$ is negligible.

We have also investigated the Goldstone effect at $\beta=5.1$ and 5.2. Both values are below the deconfinement transition point $\beta_{d}=5.236(3)$. In Fig. 7 we show the
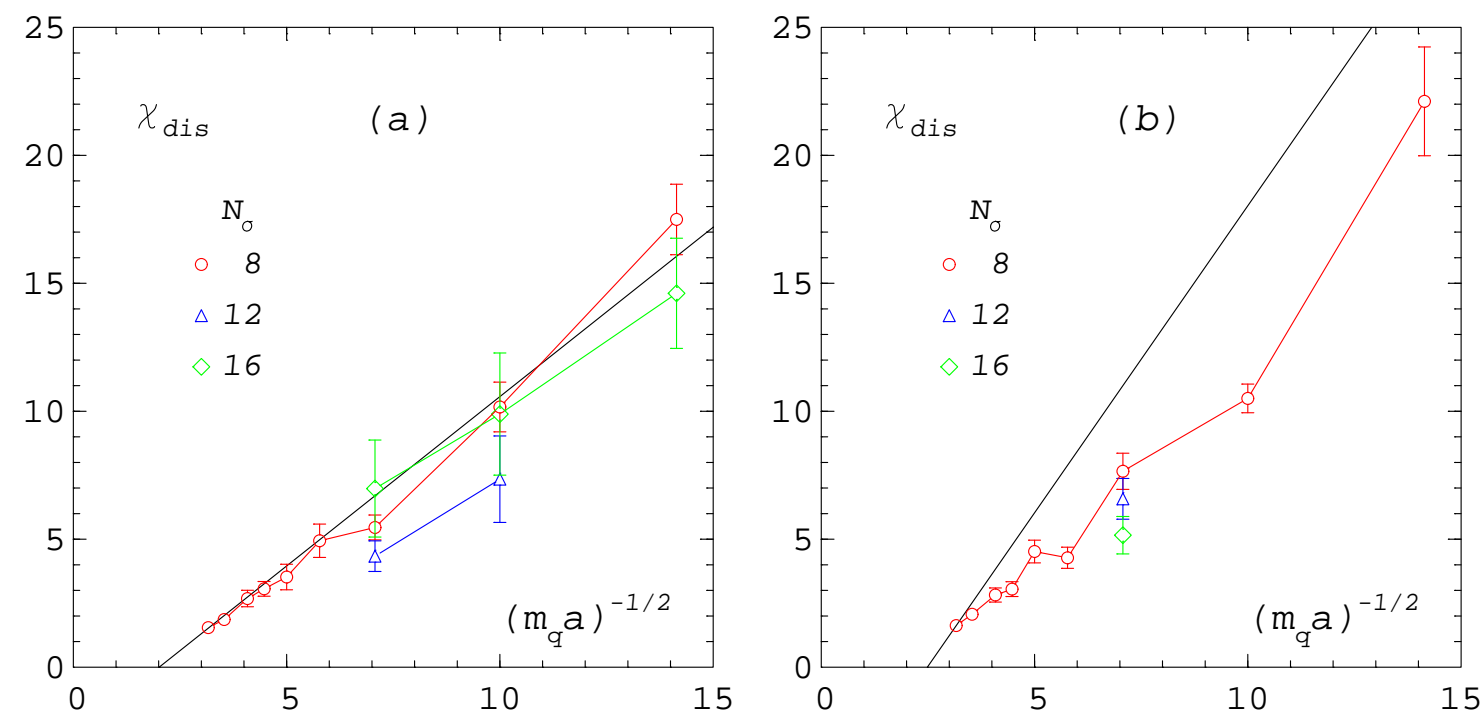

Figure 6: The chiral susceptibility $\chi_{\text {dis }}$ versus $\left(m_{q} a\right)^{-1 / 2}$ for $\beta=5.6$ (a) and $\beta=5.65$ (b). The straight lines correspond to Eq. (35) with the parameters from Table 6. 

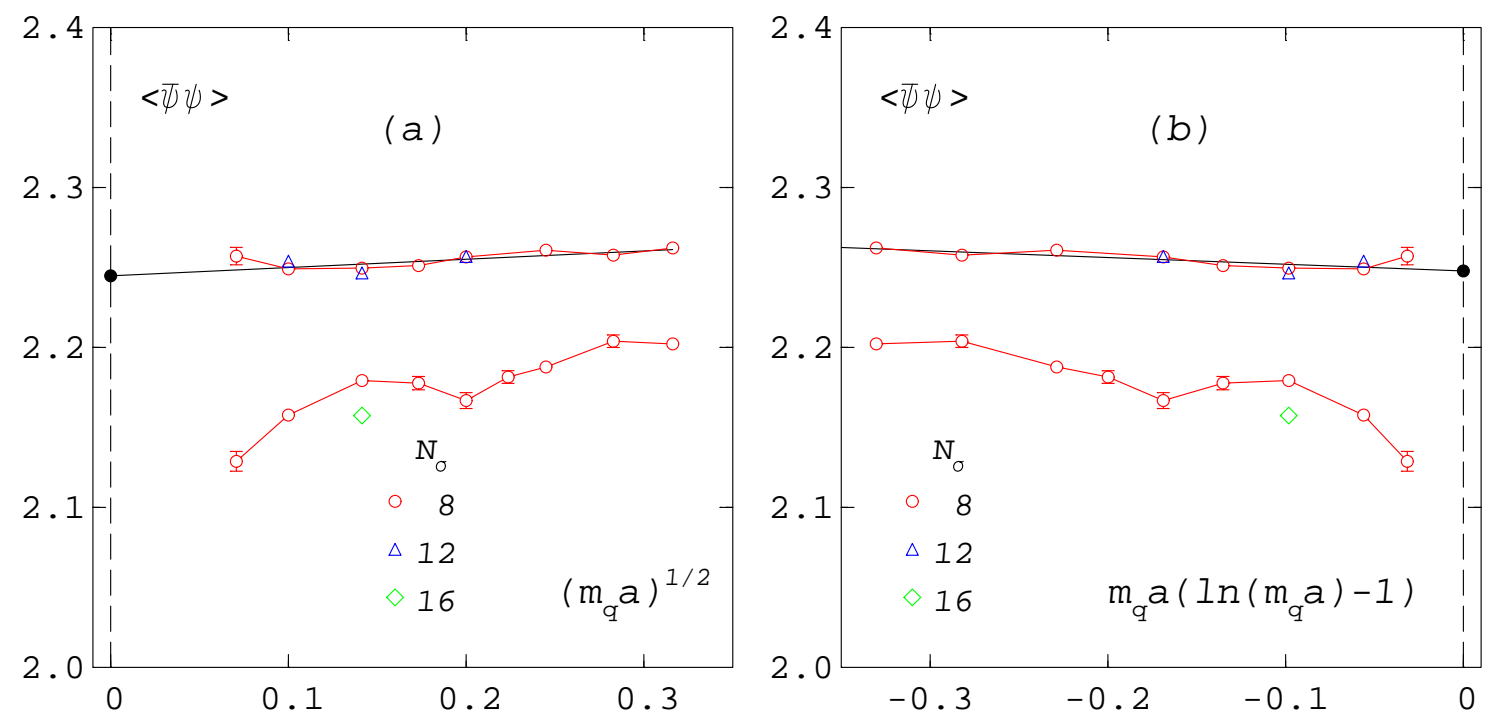

Figure 7: The chiral condensate $\langle\bar{\psi} \psi\rangle$ for $\beta=5.1$ (upper points) and $\beta=5.2$ (lower points) versus $\left(m_{q} a\right)^{1 / 2}$ (a) and versus $m_{q} a\left(\ln \left(m_{q} a\right)-1\right)(\mathrm{b})$. The straight lines are fits to ansatz (33) in (a) and to ansatz (34) in (b).

data for the chiral condensate. Due to the nearby deconfinement transition the data for $\beta=5.2$ are still too noisy to enable an analysis. At $\beta=5.1$, on the other hand, the data are nearly independent of the mass and fits to ansatz (33), Fig. 7(a), and ansatz (34), Fig. 7(b), are both possible, without favouring one of them.

As mentioned already, the verification of the Goldstone ansatz (33) leads to an estimate of the chiral condensate on the coexistence line. In the vicinity of the critical point we expect the following critical behaviour

$$
\langle\bar{\psi} \psi\rangle\left(\beta<\beta_{c}, m_{q} a=0\right)=B\left(\beta_{c}-\beta\right)^{\beta_{m}}=\left(-\beta_{r}\right)^{\beta_{m}}
$$

Here, $\beta_{m}$ is the magnetic critical exponent (called $\beta$ in the case of the $O(N)$ spin models). From the second part of Eq. (36) it is clear that the critical amplitude $B$ and the normalization $\beta_{0}$ are related by $\beta_{0}=B^{-1 / \beta_{m}}$. In order to determine $B$ we have to evaluate the estimates of the chiral condensate at $m_{q} a=0$ from Table 6 . These data are shown in Fig. 8. Of course, at larger values of $\beta_{c}-\beta$ corrections to the leading term are expected to contribute. We have therefore first tried fits to Eq. (36) without the last three points, using $\beta_{m}$ from the $O(2)$ and $O(6)$ models, or as a free parameter. In all three cases the $\chi^{2} /$ d.o. $f$. is of the order 16-20 when the point at $\beta=5.6$ is included in the fit. Most probably, due to the vicinity of the critical point, the extrapolation of the chiral condensate to $m_{q} a=0$ at $\beta=5.6$ is not as reliable as at the higher $\beta$-values. Discarding that point, we obtain excellent and essentially coinciding fits, if $\beta_{m}$ is treated as a free parameter and for $\beta_{m}(O(2))$. The fit is not as good, if $\beta_{m}$ from the $O(6)$ model is used. We have also made fits 


\begin{tabular}{|c|c|c|c||c|c|}
\hline$B$ & $\beta_{m}$ & $b_{1}$ & $\theta$ & $\left(\beta_{c}-\beta\right)$-range & $\chi^{2} /$ d.o.f. \\
\hline $2.10(02)$ & $O(2)$ & - & - & $0.074-0.174$ & 0.075 \\
\hline $2.43(02)$ & $O(6)$ & - & - & $0.074-0.174$ & 4.100 \\
\hline $2.06(12)$ & $0.338(30)$ & - & - & $0.074-0.174$ & 0.012 \\
\hline $2.04(06)$ & $O(2)$ & $1.39(86)$ & $1.98(68)$ & $0.074-0.324$ & 2.21 \\
\hline $2.42(03)$ & $O(6)$ & $2.4(5.3)$ & $3.4(2.1)$ & $0.074-0.324$ & 4.06 \\
\hline
\end{tabular}

Table 7: The parameters of the fits according to Eqs. (36) and (37).

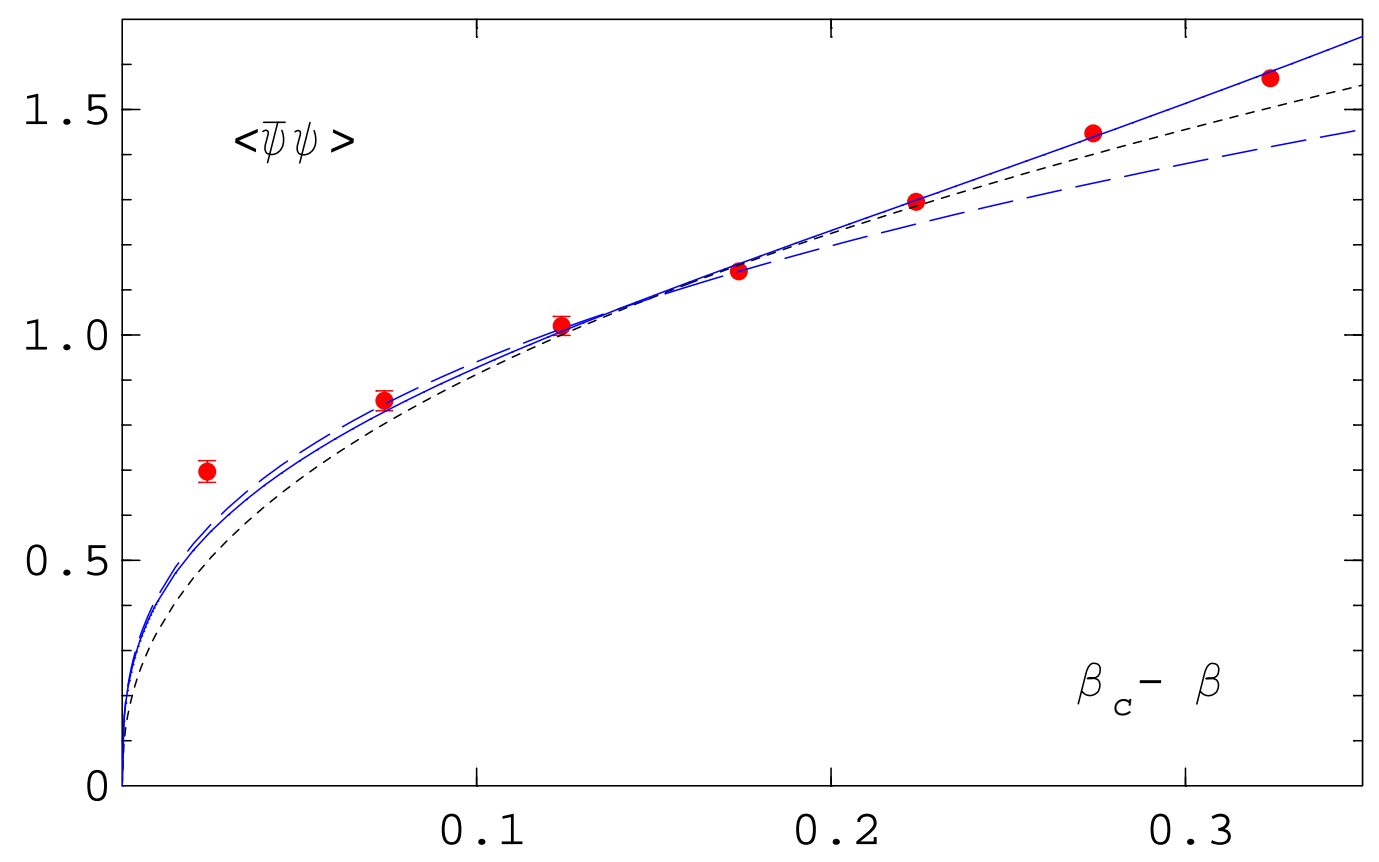

Figure 8: The chiral condensate $\langle\bar{\psi} \psi\rangle$ at $m_{q} a=0$ from Table 6 (filled circles) as a function of $\beta_{c}-\beta$. The dashed lines are fits to Eq. (36) using the points in the range $0.05<\beta_{c}-\beta<0.2$ with $\beta_{m}$ from $O(2)$ (long dashes) and $O(6)$ (short dashes). The solid line is a fit to Eq. (37) with $\beta_{m}$ fixed to the $O(2)$-value.

with an ansatz including a correction-to-scaling term

$$
\langle\bar{\psi} \psi\rangle\left(\beta<\beta_{c}, m_{q} a=0\right)=B\left(\beta_{c}-\beta\right)^{\beta_{m}}\left[1+b_{1}\left(\beta_{c}-\beta\right)^{\theta}\right],
$$

and all available points apart from the first one. In Fig. 8 we show the result for $\beta_{m}$ fixed to the $O(2)$-value as a solid line. Here, the correction exponent is rather large, $\theta \approx 2$. A similar fit with $\beta_{m}(O(6))$ is again worse and leads to large errors in the parameters. A fit to Eq. (37), where also $\beta_{m}$ is free, is very unstable and leads to a negative $\beta_{m}$. Details of our fits are listed in Table 7 . 


\subsection{Scaling behaviour of the chiral condensate}

In the last subsections we have seen that the chiral condensate data in the close vicinity of the chiral phase transition are compatible with $O(2)$ and, to a lesser degree, also $O(6)$ critical behaviour. In the following we want to compare the universal scaling function for aQCD, $f=\langle\bar{\psi} \psi\rangle m^{-1 / \delta}$, to those of the spin models, $f_{G}=M h^{-1 / \delta}$. In Fig. 9 we show the data for $\langle\bar{\psi} \psi\rangle m^{-1 / \delta}$ as a function of the scaling variable $z=\beta_{r} / m^{1 / \Delta}$ with the critical exponents for the $O(2)$ (a) and $O(6)$ (b) models from Table 1 . The used normalizations $\left(m_{q} a\right)_{0}$ and $\beta_{0}$ are shown in Table 8 . The mass normalization was calculated from the amplitude $d_{c}$ determined in subsection 4.1. The normalization $\beta_{0}$ can be deduced from the amplitude $B$ computed in the last subsection. Alternatively, one may fix the derivative of the scaling function at the critical point to the one of the corresponding $O(N)$ model. This is what we have done for the plots. The derivative is given by

$$
f^{\prime}(0)=d_{c}^{1} \beta_{0} d_{c}^{\left(1 / \beta_{m}-1\right)} .
$$

The derivatives of the $O(2)$ and $O(6)$ scaling functions can be found from the respective parametrizations in Refs. [23, 27]. Choosing this possibility we obtain $B=2.06$ for $O(2)$ and $B=2.27$ for $O(6)$. That is, for $O(2)$ we find a $B$-value which is in agreement with the $O(2)$ and free fit results from Table 7 , whereas the $O(6)$ fits lead to somewhat higher $B$-values. In Fig. 9 we have plotted only the data from the range $5.3 \leq \beta \leq 5.9$, at $\beta=5.3$ only the data for $m_{q} a \leq 0.03$. All other data are either distorted by the deconfinement transition or too far outside the critical region.

We observe in Fig. 9 (a) that the data scale very well with $O(2)$ exponents for small $|z|$ and agree there also with the $O(2)$ scaling function $f_{G}(z)$. For decreasing $z<0$ the data for different $\beta$ start to deviate from each other. A similar effect was found for the $O(2)$ data [23] and is characteristic for corrections to scaling. At $z>0$ the data scale well apart from the data for $m_{q} a=0.005$. This may be due to the presence of finite size effects, though the few data with low statistics which we have from the larger lattices coincide inside the error bars with the $8^{3} \times 4$ data. In Fig. 9 (b), where we show the data using $O(6)$ parameters, the situation is somewhat different. Here, the data scale very well apart from the neighbourhood of the critical

\begin{tabular}{|c||c|c||c|c|c|c|}
\hline & $\left(m_{q} a\right)_{0}$ & $\beta_{0}$ & $d_{c}$ & $d_{c}^{1}$ & $f_{G}^{\prime}(0)$ & $B$ \\
\hline$O(2)$ & 0.0093 & 0.126 & 2.66 & -0.366 & -0.286 & 2.06 \\
\hline$O(6)$ & 0.0091 & 0.145 & 2.67 & -0.605 & -0.332 & 2.27 \\
\hline
\end{tabular}

Table 8: The normalization constants and the corresponding amplitudes used in the scaling plots. 

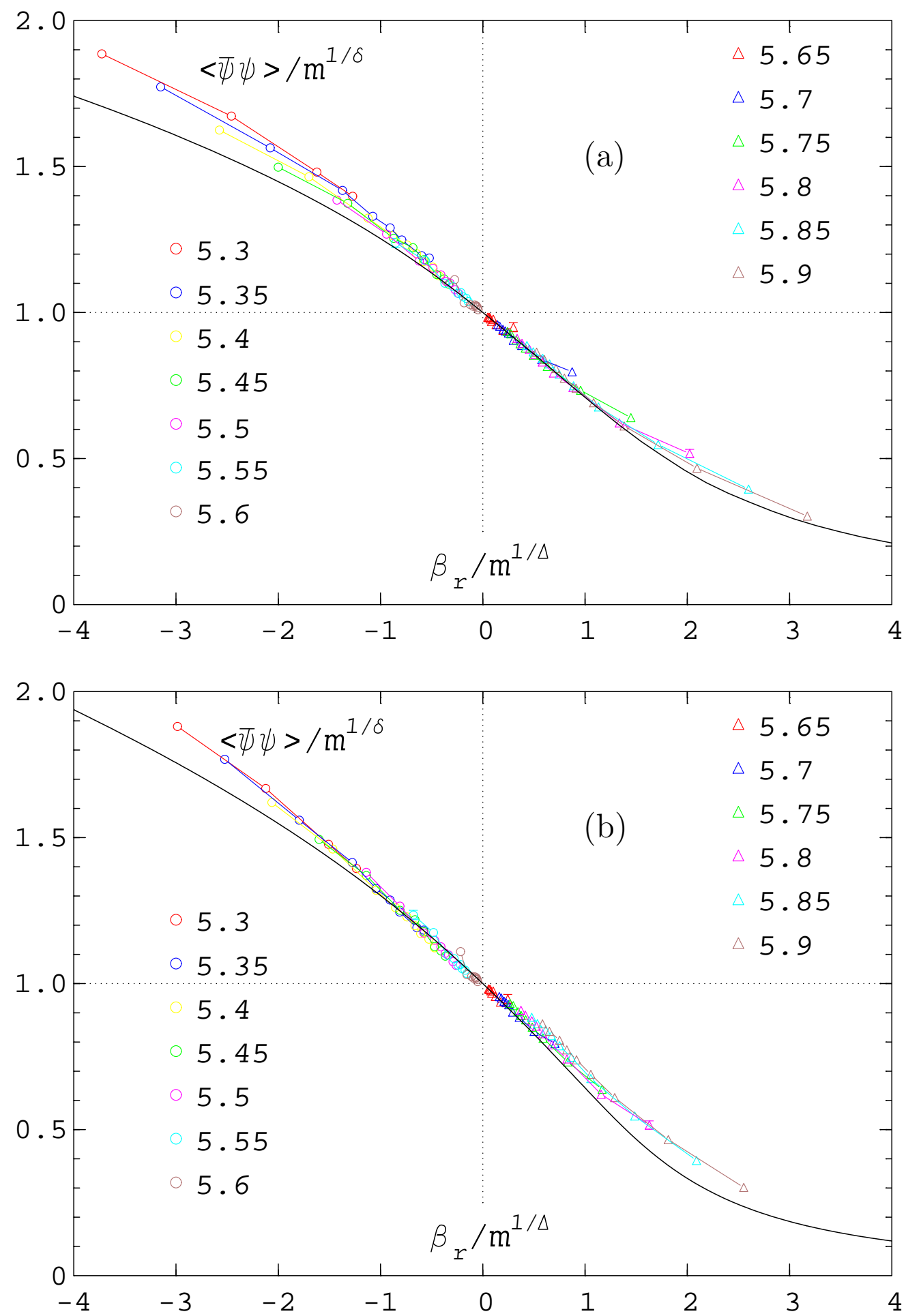

Figure 9: The scaling function $f(z)=\langle\bar{\psi} \psi\rangle / m^{1 / \delta}$ versus the scaling variable $z=$ $\beta_{r} / m^{1 / \Delta}$ with data from the $8^{3} \times 4$ lattice for $O(2)$ (a) and $O(6)$ (b) critical exponents and normalizations from Table 8 . The solid curves are the universal scaling functions from the respective $O(N)$ spin models. The data points are connected by straight lines to guide the eye, the numbers indicate the $\beta$-values of the data. 

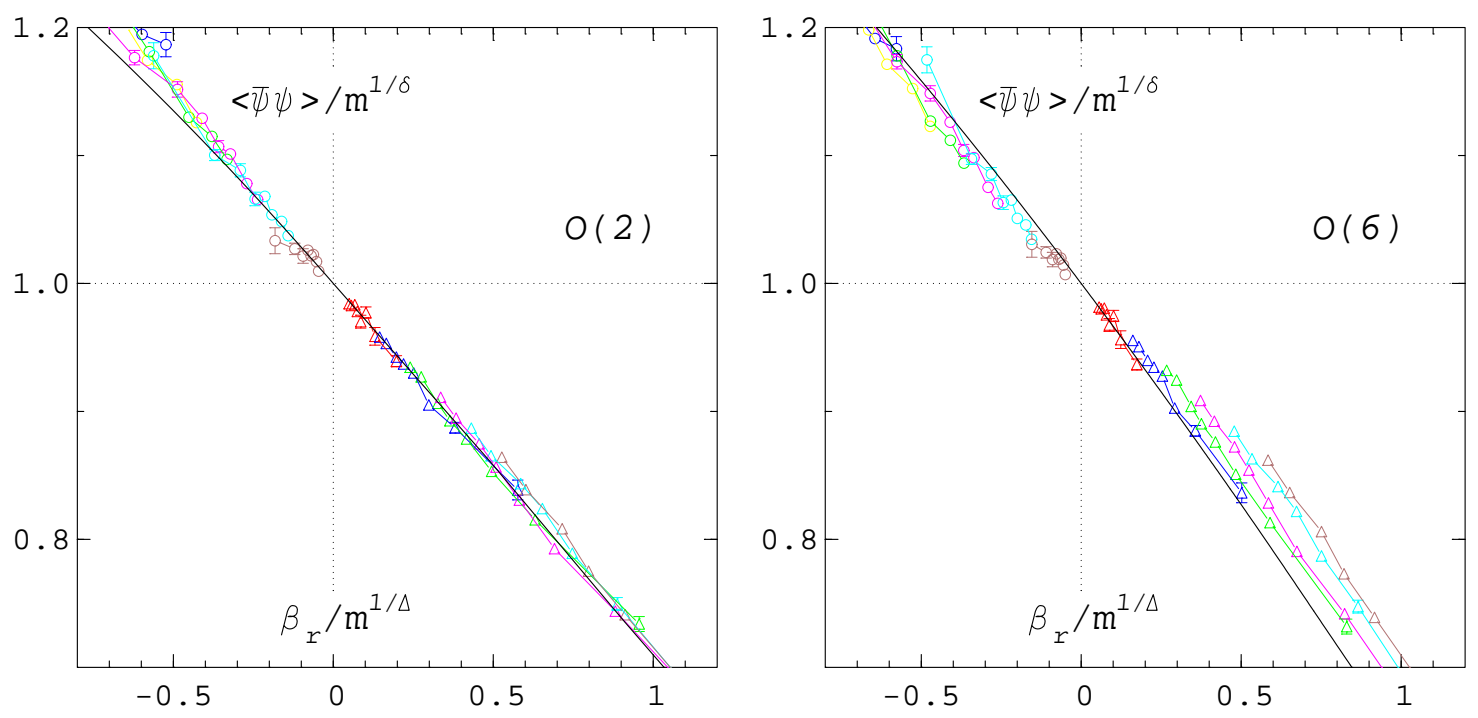

Figure 10: Comparison of the scaling behaviour of $\langle\bar{\psi} \psi\rangle / m^{1 / \delta}$ for $O(2)$ (left) and $O(6)$ (right) exponents and normalizations in the vicinity of the critical point. The notation is the same as in the previous figure. For clarity the data for $m_{q} a=0.005$ have been omitted. The black solid lines are the respective $O(N)$ scaling functions.

point where the data spread somewhat and deviate from the $O(6)$ scaling function, except very close to the normalization point $z=0$. This is in contrast to the $O(2)$ case and can be seen in more detail in Fig. 10, which shows the scaling behaviour in the close vicinity of the critical point.

As mentioned in the introduction there are first results [19] for the universality class which is expected to govern the chiral transition in the continuum limit. The universal scaling function of the class is still unknown, but estimates of two critical exponents, namely $\eta \approx 0.2, \nu \approx 1.1$ are known. The errors are still large, e.g. $\eta=0.2(1)[33]$. Due to the relation

$$
\delta=\frac{5-\eta}{1+\eta}
$$

for $d=3$ this implies $\delta=4.0(4)$, and from the considerations in subsection 4.1 and the numbers in Table 3 this entails a shift in the location of the critical point. Though we have no scaling function to compare with we can still perform a test on the scaling of the data assuming critical exponents compatible with the estimates of Ref. [19]. We have varied the exponents correspondingly and calculated each time the adequate critical point. In Fig. 11 we show the best scaling result for $\langle\bar{\psi} \psi\rangle /\left(m_{q} a\right)^{1 / \delta}$ as a function of $\left(\beta-\beta_{c}\right) /\left(m_{q} a\right)^{1 / \Delta}$ which is obtained for $\delta=4.4, \nu=1.0(1 / \Delta=0.409)$ and $\beta_{c}=5.64$. We see from Fig. 11 that the data still spread considerably around the critical point and do not indicate a definite scaling function. 


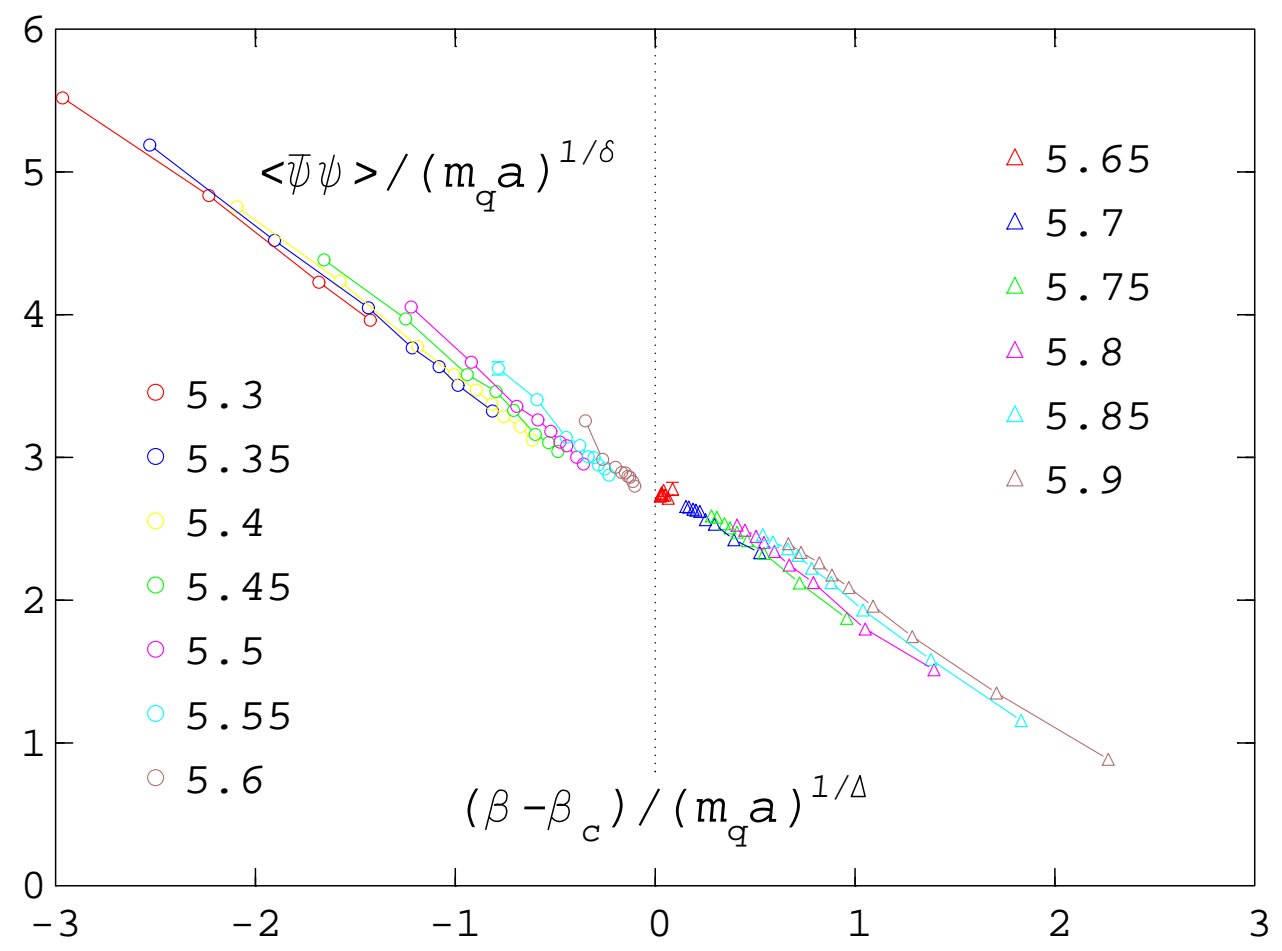

Figure 11: Scaling test for $\langle\bar{\psi} \psi\rangle /\left(m_{q} a\right)^{1 / \delta}$ with critical exponents from [19]. The data from the $8^{3} \times 4$ lattice are plotted as a function of $\left(\beta-\beta_{c}\right) /\left(m_{q} a\right)^{1 / \Delta}$. The notation is the same as in Fig. 9.

\section{$5 \quad$ Summary and Conclusions}

In this paper we have studied QCD with two flavours of staggered Dirac fermions in the adjoint representation at finite temperature. Our main objective was the investigation of the chiral transition and the determination of the associated universality class. For ordinary QCD with two staggered fermions this issue is still under discussion and may be difficult to decide because the deconfinement transition coincides with the chiral transition. In contrast to QCD the corresponding transitions of aQCD occur at two distinct critical temperatures and therefore allow to study the transitions separately. A major prerequisite of any scaling test on critical behaviour is the exact location of the critical point. In order to achieve that goal and also to obtain sufficient data for the tests we have extended the existing data base for aQCD supplied by the work of Karsch and Lütgemeier [16].

The critical point of the chiral transition was then determined using several strategies. Whereas the peak positions of the chiral susceptibility provide only first hints on a $\beta_{c}$-value below 5.79 - the result of Ref. [16] - the evaluation of the mass dependence of $\langle\bar{\psi} \psi\rangle$ leads definitely to an interval $5.6 \leq \beta_{c} \leq 5.7$ and a value $0.19 \leq 1 / \delta \leq 0.28$ for the citical exponent of $\langle\bar{\psi} \psi\rangle$ on the critical line. The known values of $\delta$ for the $O(N)$ spin models are all close 4.8 , that is $1 / \delta \approx 0.21$. The 


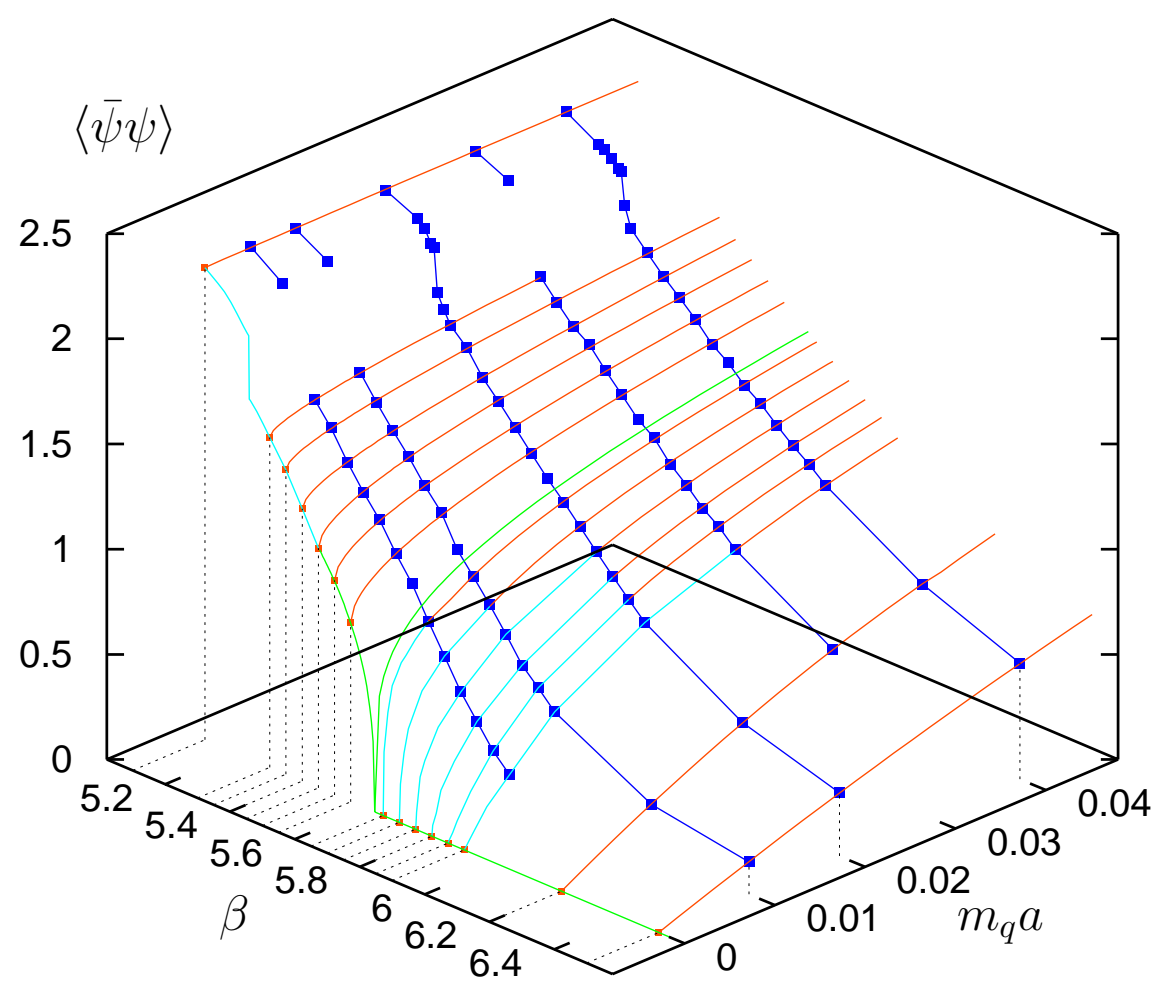

Figure 12: Three-dimensional plot of the chiral condensate $\langle\bar{\psi} \psi\rangle$ for all $\beta$-values und $m_{q} a \leq 0.04$. The data are from the $8^{3} \times 4$ lattice. The dark blue lines connect the same masses, the green ones show fits to the critical behaviour on the coexistence line and the critical line. The red lines are fits of the mass dependencies at fixed $\beta$ and the light blue lines show the expected behaviour in regions where we do not have enough data. The dotted lines show the hights and indicate the $\beta$-values.

value $\delta=4.0(4)$ of the new universality class proposed by Ref. [19] is however also compatible with the found $1 / \delta$-interval. In the following we improved on our $\beta_{c^{-}}$ estimate by including the next term in the scaling ansatz, using the exponents of the $O(2)$ and $O(6)$ model and later also the ones from Ref. [19]. As it turned out both spin models lead to the same rather accurate value $\beta_{c}=5.624(2)$. This value and the respective value $\beta_{d}=5.236(3)$ [16] of the deconfinement transition yield, via the two-loop beta function, the ratio $T_{c} / T_{d}=7.8(2)$.

A further aim of our paper was the verification of the Goldstone effect which is expected to control the mass dependence of the chiral condensate below the chiral transition temperature. In the three-dimensional $O(N)$ models the effect is even detectable in the critical region close to $T_{c}$ due to its influence on the universal scaling function $f_{G}(z)$. We have evaluated the chiral condensate data at fixed $\beta<\beta_{c}$ in the same manner as it was done for the magnetization of the three-dimensional $O(N)$ models and find a completely analogous behaviour, that is aQCD acts as an effective three-dimensional $O(N)$ spin model in its chirally broken phase. However, 
close to the deconfinement transition this behaviour is disturbed and $\langle\bar{\psi} \psi\rangle$ develops a gap. Below $\beta_{d}$, at $\beta=5.1,\langle\bar{\psi} \psi\rangle$ becomes a smooth, though only slightly varying function of $m_{q} a$, which could as well be interpreted as the behaviour of an effective four-dimensional theory.

Using the obtained values for the critical point and the critical amplitudes for the normalization of the scales we have carried out scaling tests and comparisons to the scaling functions of the $O(2)$ and $O(6)$ models. With the $O(2)$ critical exponents the data are scaling convincingly in the direct neighbourhood of the critical point, that is for small values of $|z|$ and they coincide there with the $O(2)$ scaling function. For larger values of $(-z)$ the data show - like in the original non-linear $\sigma$-model increasing deviations, which are typical for corrections to scaling. Using the $O(6)$ parameters for the scaling test seems at first sight to lead to scaling of the data in a larger region of $z$. A closer inspection in the vicinity of the critical point, where the $O(2)$ test works, shows however, that the data spread there and deviate somewhat from the $O(6)$ scaling function. This observation is confirmed by the calculation of the amplitude $B$ from the derivative of the scaling function: it is in better agreement with the value found from the Goldstone effect for $O(2)$ than for $O(6)$. We have also carried out scaling tests with the critical exponents calculated in Ref. [19] for the universality class of the continuum limit. Our lattice data do not yet support the proposed class.

In conclusion, our findings have led to a consistent picture of the behaviour of aQCD from below the deconfinement transition to well above the chiral transition. We have found evidence for a second order chiral transition at $\beta_{c}=5.624(2)$, compatible with the $3 d O(2)$ universality class. The expected $3 d$ Goldstone effect in the intermediate phase $\beta_{d}<\beta<\beta_{c}$, which was already noted in Ref. [16], was fully confirmed. The same applies to the first order deconfinement transition at $\beta_{d}=5.236(3)$. Our results are visualized in Fig. 12, where we show the chiral condensate data at small mass values together with fits based on our understanding of the behaviour of aQCD at finite temperature.

\section{Acknowledgments}

We thank Frithjof Karsch and Martin Lütgemeier for many discussions of their work on QCD with adjoint fermions and the use of their programs and data. We are also grateful to Edwin Laermann for clarifying comments on the chiral susceptibility and on algorithmic problems. Our work was supported by the Deutsche Forschungsgemeinschaft under Grants No. FOR 339/2-1 and No. En 164/4-4. 


\section{References}

[1] R. D. Pisarski and F. Wilczek, Phys. Rev. D 29 (1984) 338.

[2] F. Wilczek, Int. J. Mod. Phys. A 7 (1992) 3911 [Erratum-ibid. A 7 (1992) 6951].

[3] K. Rajagopal and F. Wilczek, Nucl. Phys. B 399 (1993) 395 [hep-ph/9210253].

[4] Y. Iwasaki, K. Kanaya, S. Kaya and T. Yoshie, Phys. Rev. Lett. 78 (1997) 179 [hep-lat/9609022].

[5] A. Ali Khan et al. [CP-PACS Collaboration], Phys. Rev. D63 (2001) 034502 [hep-lat/0008011].

[6] F. Karsch, Phys. Rev. D 49 (1994) 3791 [hep-lat/9309022].

[7] F. Karsch and E. Laermann, Phys. Rev. D 50 (1994) 6954 [hep-lat/9406008].

[8] S. Aoki et al. [JLQCD Collaboration], Phys. Rev. D 57 (1998) 3910 [heplat/9710048].

[9] E. Laermann, Nucl. Phys. Proc. Suppl. B 60A (1998) 180.

[10] C. W. Bernard et al. [MILC Collaboration], Phys. Rev. D 61 (2000) 054503 [hep-lat/9908008].

[11] J. Engels, S. Holtmann, T. Mendes and T. Schulze, Phys. Lett. B 514 (2001) 299 [hep-lat/0105028].

[12] J. B. Kogut and D. K. Sinclair, Simulating lattice QCD at finite temperature and zero quark mass, hep-lat/0408003.

[13] S. Chandrasekharan and C. G. Strouthos, Phys. Rev. D 69 (2004) 091502 [heplat/0401002].

[14] M. D'Elia, A. Di Giacomo and C. Pica, Two flavor QCD and confinement, hep-lat/0503030.

[15] J. B. Kogut, J. Polonyi, H. W. Wyld and D. K. Sinclair, Phys. Rev. Lett. 54 (1985) 1980.

[16] F. Karsch and M. Lütgemeier, Nucl. Phys. B 550 (1999) 449 [hep-lat/9812023].

[17] M. E. Peskin, Nucl. Phys. B 175 (1980) 197.

[18] A. Smilga and J. J. M. Verbaarschot, Phys. Rev. D 51 (1995) 829 [hepth/9404031].

[19] F. Basile, A. Pelissetto and E. Vicari, JHEP 0502 (2005) 044 [hep-th/0412026]. 
[20] M. Lütgemeier, Phasenübergänge in der QCD mit fundamentalen und adjungierten Quarks, Ph. D. Thesis, Universität Bielefeld, November 1998.

[21] K. Bitar, A. D. Kennedy, R. Horsley, S. Meyer and P. Rossi, Nucl. Phys. B 313 (1989) 377.

[22] A. Pelissetto and E. Vicari, Phys. Rept. 368 (2002) 549 [cond-mat/0012164] .

[23] J. Engels, S. Holtmann, T. Mendes and T. Schulze, Phys. Lett. B 492 (2000) 219 [hep-lat/0006023].

[24] D. Toussaint, Phys. Rev. D 55 (1997) 362 [hep-lat/9607084].

[25] J. Engels and T. Mendes, Nucl. Phys. B 572 (2000) 289 [hep-lat/9911028].

[26] J. Engels, L. Fromme and M. Seniuch, Nucl. Phys. B 675 (2003) 533 [heplat/0307032].

[27] S. Holtmann and T. Schulze, Phys. Rev. E 68 (2003) 036111 [hep-lat/0305019].

[28] J. Zinn-Justin, Quantum Field Theory and Critical Phenomena, Clarendon Press, Oxford, 3rd Edition 1996.

[29] D. J. Wallace and R. K. P. Zia, Phys. Rev. B 12 (1975) 5340.

[30] R. Anishetty, R. Basu, N. D. Hari Dass and H. S. Sharatchandra, Int. J. Mod. Phys. A 14 (1999) 3467 [hep-th/9502003].

[31] J. Engels, S. Mashkevich, T. Scheideler and G. Zinovjev, Phys. Lett. B 365 (1996) 219 [hep-lat/9509091].

[32] W. E. Caswell, Phys. Rev. Lett. 33 (1974) 244.

[33] E. Vicari, private communication. 